\title{
Temperature dynamics of the electronic structure in dilute Bi-Sn alloys
}

\author{
A. S. Fedotov, ${ }^{1,{ }^{*}}$ V. G. Shepelevich, ${ }^{1}$ I. A. Svito, ${ }^{1}$ and V. A. Sivakov ${ }^{2}$ \\ ${ }^{1}$ Belarusian State University, 4 Nezalezhnasci av., Minsk 220030, Belarus \\ ${ }^{2}$ Institute of Photonic Technology, Albert Einstein Str. 9, D-07745 Jena, Germany
}

(Received 3 February 2017; published 20 February 2018)

\begin{abstract}
We study the changes of Bi electronic structure near $T$ and $L$ points of the Brillouin zone caused by doping with $\mathrm{Sn}$ (concentrations $\leqslant 0.08$ at.\%). Hall coefficient and magnetoresistance measurements (under magnetic field up to $8 \mathrm{~T}$ ) enabled calculation of magnetoconductivity tensor components. The usage of quantitative mobility spectrum analysis together with the isotropic approximation for band structure allowed the estimation of Fermi level position at temperatures 10-300 K. The results have shown that Sn doping shifts the Fermi level down on the energy scale at the $L$ point (in all temperature range) and at the $T$ point under (primarily at low temperatures), leading to the decrease of band overlap.
\end{abstract}

DOI: 10.1103/PhysRevB.97.075204

\section{INTRODUCTION}

Semimetals [1] and narrow-gap semiconductors [2] have found numerous applications in modern electronics as they combine high mobility of charge carriers [1] and low-energy electronic transitions [3]. The recent progress in development of thermoelectric devices [4-6], THz emitters [7,8], and infrared detectors $[3,9]$ justifies the need for understanding the influence of various factors (like temperature and doping) on electronic states in this class of materials. However, current knowledge of temperature, doping, and morphology effects on those properties needs further improvement, especially for the Bi-based materials. The modern advantages in methods of galvanomagnetic measurements processing [10-12] open the new prospectives for better understanding the doping effect on charge carrier parameters in a wide temperature range for Bi-based compositions. We explore these new possibilities in order to elucidate the very electronic band changes of semimetallic samples under doping and temperature influence.

Bismuth is a canonical semimetal with highly anisotropic Fermi surface originating from a complicated unit cell [13-17], resulting in the electronic structure $[14,15,18-20]$ schematically presented in Fig. 1 . The band at the $L$ point is a source of light electrons (with effective mass $m_{n} \approx 10^{-2}$ of free electron mass $m_{0}$ ), the band at the $T$ point is a source of heavier holes (with $m_{p} \approx 10^{-1} m_{0}$ ). Fermi level $E_{F}$ is positioned close to bands' extrema [19-21]: the distance between the $E_{F}$ and $L$-point band minimum is $\zeta_{n}=27 \mathrm{meV}$, the distance between the $E_{F}$ and $T$-point band maximum is $\zeta_{p}=11 \mathrm{meV}$ at $4 \mathrm{~K}$ (see Fig. 1). The electronic band structure is characterized by small indirect overlap $E_{o}=\zeta_{n}+\zeta_{p}$ between bands as well as nonparabolic dispersion law at the $L$ point $[21,22]$. The parameters $\zeta_{n}, \zeta_{p}, E_{o}$ and band gap $E_{g}$ exhibit strong temperature dependencies growing approximately three times from liquid helium temperature (LHT) to room temperature (RT) $[19,20,23]$.

\footnotetext{
*fedotov.alehandro@gmail.com
}

Nowadays, despite much effort devoted to Bi investigation, the influences of doping and temperature on electronic states require better understanding. For example, the effects of $\mathrm{Sn}$ doping [24-27] are known mostly qualitatively for temperatures above LHT. Doping with Sn turns Bi to $p$ type at low temperatures returning to mixed conductivity at RT. The influence of doping for small concentrations $\left(\lesssim 10^{-1}\right.$ at.\%) can be described as result of Fermi level shift deeper into hole band at the $T$ point [25,27]. There were reports on light holes in doped samples [25,26] with effective mass similar or less than mass $m_{n}$ of $L$-point electrons. Concentration dependence of Fermi level $\zeta_{p}$ position for $\mathrm{Bi}_{100-x} \mathrm{Sn}_{x}, x<$ 0.1 at.\%, was systematically investigated by Para [27] and Nikolaeva [28] by analysis of Shubnikov-de Haas oscillations in single-crystalline wires. It is reasonable to expect significant temperature dependence of band parameters in analog to nondoped Bi, but the Shubnikov-de Haas effect is observable only at low temperatures.

Several electrical methods are suitable for charge carrier characterization. Methods based on cyclotron resonance [29,30] and Shubnikov-de Haas oscillations [16,31] require low temperatures and very high quality of samples. The galvanomagnetic measurements $[23,32,33]$ of the Hall effect and magnetoresistance allowed broader range of temperatures, but usually need achievement of weak or strong magnetic field limits that is difficult in case of very low or high carrier mobility. The modern quantitative mobility spectrum analysis (QMSA) methods [10-12,34-37] use information in all available ranges of magnetic fields and require minimum external input parameters for the computational procedure. This makes QMSA a very promising tool for investigation of electronic states in diluted $\mathrm{Bi}_{100-x} \mathrm{Sn}_{x}$ alloys.

Therefore, despite a lot of measurements and quantitative investigations, the movement of Fermi level is practically unknown in diluted $\mathrm{Bi}_{100-x} \mathrm{Sn}_{x}(x<0.08$ at.\%) compounds at temperatures higher than $10 \mathrm{~K}$. In this paper, we report the temperature dependence of Fermi level position relative to the $T$ - and $L$-point band extrema in the range $10-300 \mathrm{~K}$. We have found that the band overlap $E_{o}$ decreases with 
(a)

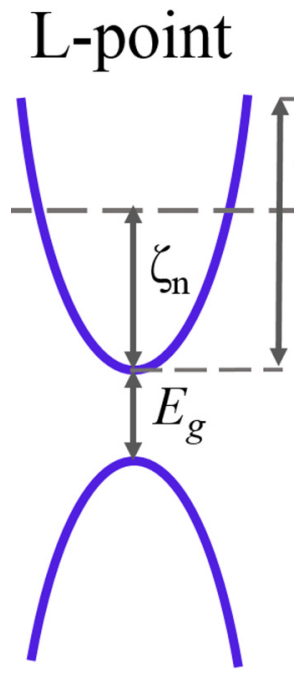

T-point

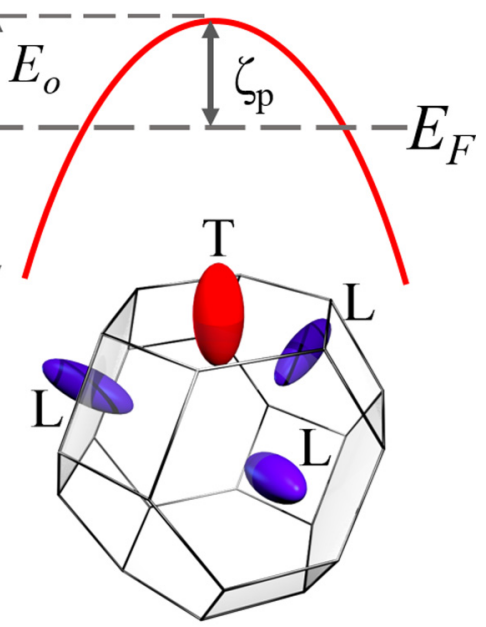

(b)
FIG. 1. Simplified band structure of Bi (a) and Fermi surface (b).

doping and disappears at RT for $x=0.08$ at.\%. Additionally, it was shown that temperature dependence of Fermi level can be qualitatively described at low temperatures by means of Sommerfeld expansion.

\section{EXPERIMENTAL DETAILS}

Alloys of $\mathrm{Bi}_{100-x} \mathrm{Sn}_{x}, 0 \leqslant x \leqslant 0.08$ at.\%, were prepared by melt spinning $[38,39]$ of high-purity initial materials. Thickness of samples showed less than $2 \%$ fluctuations. Preferred crystalline orientations partially compensate each other. For detailed information on structure, see Appendix A.

Hall coefficient $R_{H}$ and magnetoresistance $\rho(B)$ were measured in the temperature range 10-300 K under magnetic fields up to $8 \mathrm{~T}$ using a close-cycled measuring system by Cryogenic Ltd. Details on the experiment and raw data processing are given in Appendix B.

\section{CONDUCTIVITY TYPE CHANGE}

As experiments showed, the Hall coefficient $R_{H}$ is positive in doped $\mathrm{Bi}_{100-x} \mathrm{Sn}_{x}$ at LHT and switches to negative with the increase of temperature. The sweeping out magnetic field increases the temperature $T_{\text {switch }}$ at which $R_{H}$ changes its sign. The curves separating regions of positive and negative $R_{H}$ are given in Fig. 2 for different $\mathrm{Sn}$ concentrations $x$. The $T_{\text {switch }}$ moves to the higher temperatures with the increase of $x$ (Fig. 3) indicating the suppression of the electron's contribution by doping. Magnetic field reverses the switch of $R_{H}$ sign and returns it to positive values (Fig. 2). Therefore, in doped samples, average mobility of electrons is still much higher than mobility of holes, so magnetic field drags electrons in the first place decreasing its contribution to charge transport.

The temperature dependencies of relative magnetoresistance (rMR) under magnetic field $8 \mathrm{~T}$ are given in Fig. 3. The rMR of Bi (1) at LHT is very high (of $10^{3} \%-10^{5} \%$ ) and monotonously decreases with temperature. The rMR

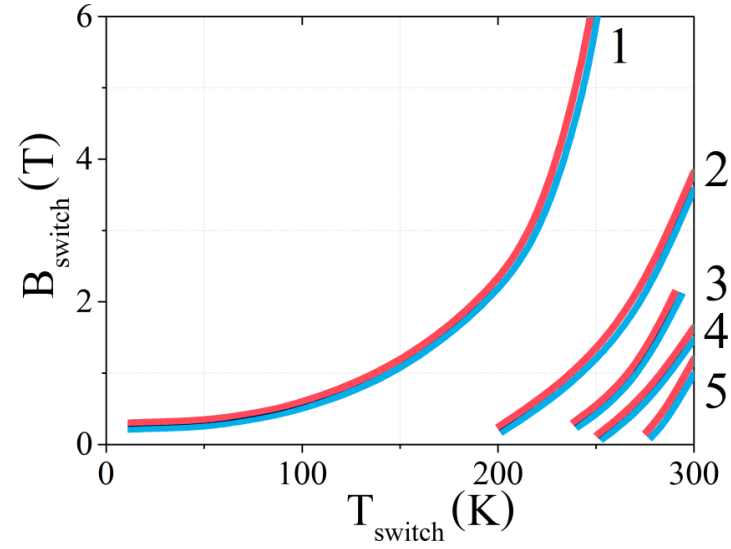

FIG. 2. Separating curves between regions of positive (red side) and negative (blue side) Hall coefficient for $\mathrm{Bi}_{100-x} \mathrm{Sn}_{x}, x$ (at.\%): 1 (0.01), 2 (0.02), 3 (0.04), 4 (0.06), 5 (0.08).

temperature dependencies of doped samples (2)-(6) are not monotonous. The well-known expression [23,33,40,41] for rMR establishes the square dependence on the carrier's mobility: $\Delta \rho(B) / \rho(0)=C_{1} \frac{p \mu_{p}^{2}+n \mu_{n}^{2}}{p \mu_{p}+n \mu_{n}}+C_{2} \frac{\left(p \mu_{p}^{2}+n \mu_{n}^{2}\right)^{2}}{\left(p \mu_{p}+n \mu_{n}\right)^{2}}\left(C_{1}, C_{2}\right.$ are constants). Therefore, small numbers of high-mobility carriers can significantly boost rMR. This confirms the activation of light electrons with temperature in doped samples. The temperature $T_{g r}$, where rMR starts to rise with the $T$, increases with the growth of Sn concentration $x$. That means reducing electron numbers with $x$ (Fig. 3).

The detailed discussion of galvanomagnetic experiment results and additional arguments for usage of QMSA are given in Appendix C.

\section{TEMPERATURE DEPENDENCIES OF ELECTRONIC CHARACTERISTICS}

The charge carrier characteristics were obtained using QMSA implementation described in Appendix D. The temperature dependencies of electron mobilities $\mu_{n}$ and

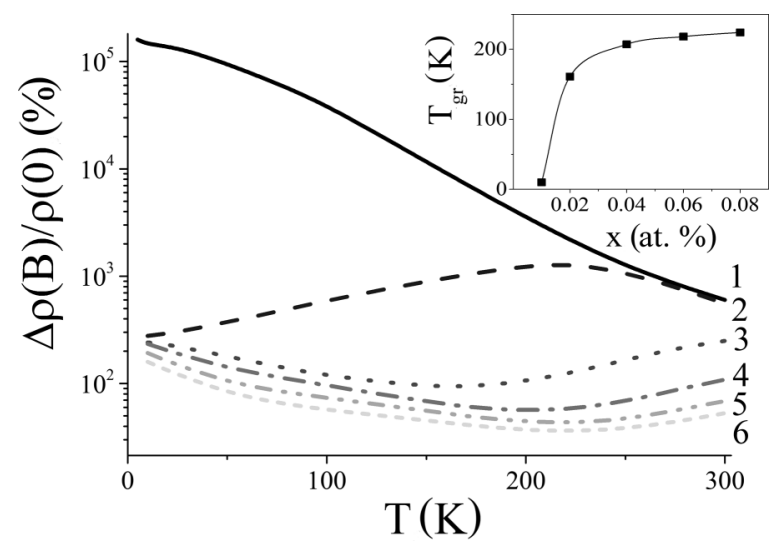

FIG. 3. Temperature dependence of relative magnetoresistance $\Delta \rho(B) / \rho(0)$ under field $B=8 \mathrm{~T}$ for various concentrations $x$ (at. \%): 1 (0) (pure Bi), 2 (0.01), 3 (0.02), 4 (0.04), 5 (0.06), 6 (0.08). Inset: the temperatures $T_{g r}$ where MR start to rise with the temperature vs Sn concentration. 


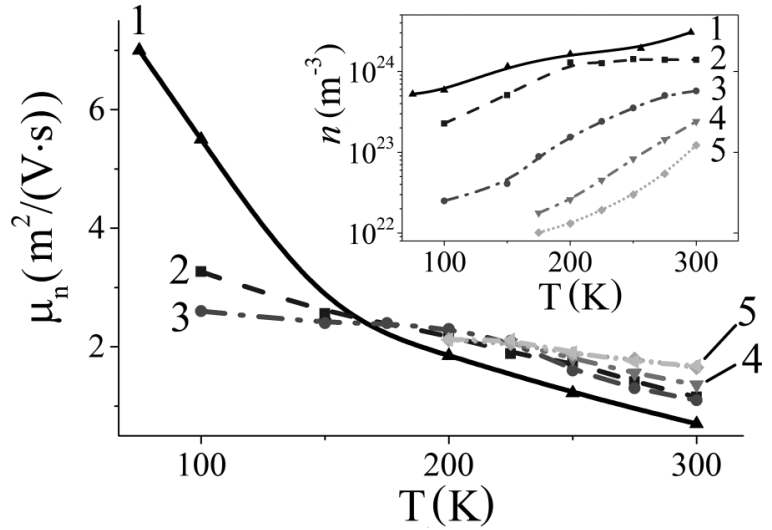

FIG. 4. Temperature dependencies of electron mobilities $\mu_{n}$ and concentrations $n$ (inset) for different Sn doping level $x: 1$ (0) (pure Bi), 2 (0.01 at.\%), 3 (0.02 at.\%), 4 (0.04 at.\%), 5 (0.06 at.\%).

concentrations $n$ for various tin concentrations $x$ are given in Fig. 4. The Sn doping significantly reduces absolute values of $\mu_{n}$ at low temperatures. The electron mobility becomes similar for all studied $x$ ranging from $T \gtrsim 175 \mathrm{~K}$. The electron concentration decreases with $x$ (inset of Fig. 4) that is in agreement with sign of $R_{H}$. From $x=0.06$ at.\% the electrons' contribution to conductivity becomes very low and undetectable by means of QMSA in the studied temperature range.

The temperature dependencies of $T$-point hole mobilities $\mu_{p}$ and concentrations $p$ for various tin concentrations $x$ are given in Fig. 5. The hole concentration $p$ rises with $x$ the most obvious at lower temperatures. While heating up to room temperature, $p$ returns to values as in nondoped $\mathrm{Bi}$. The hole concentration $p$ lowers with temperature $T$ in the samples with $x \in(0.04-0.08)$ at.\%.

The position of Fermi level relative to the band bottom in the $L$ point $\zeta_{n}$ and to the top in the $T$ point $\zeta_{p}$ can be found from the concentrations $n, p$. The nonparabolic dispersion law of electrons in the $L$ point is approximated with the Lax model [19,20], the effective mass $m_{n}$ of electrons, and the temperature dependence of band gap $E_{g}$ between $L$-point ellipsoids are

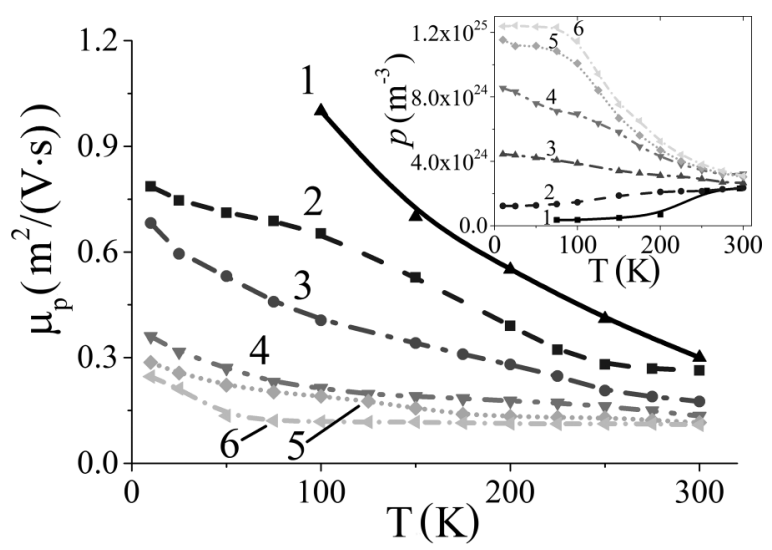

FIG. 5. Temperature dependencies of $T$-point hole mobility for different $\mathrm{Sn}$ concentration $x$ : 1 (0) (pure Bi), 2 (0.01 at.\%), (30.02 at.\%), 4 (0.04 at.\%), 5 (0.06 at.\%), 6 (0.08 at.\%).

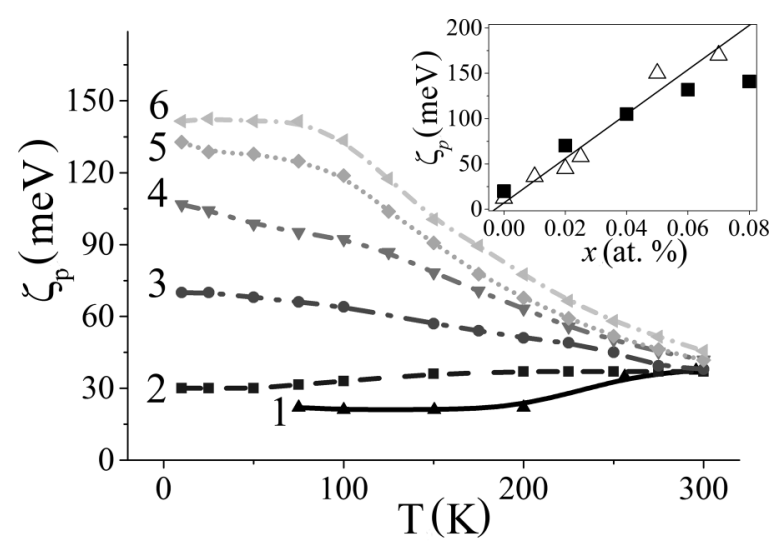

FIG. 6. Temperature dependencies of Fermi level position $\zeta_{p}$ relative to the top of band in the $T$ point for different $\mathrm{Sn}$ concentration $x: 1$ (0) (pure Bi), 2 (0.01 at.\%), 3 (0.02 at.\%), 4 (0.04 at.\%), 5 (0.06 at.\%), 6 (0.08 at.\%). Inset: doping level dependence of Fermi level position $\zeta_{p}$ relative to the top of band in $T$ point estimated from concentration integrals (black squares, $T=10 \mathrm{~K}$ ) and from literature [16,27] (white triangles, $T=4 \mathrm{~K}$ ).

taken from [20]. Therefore, the integral equation for $\zeta_{n}$ will be

$$
n=\int_{0}^{\infty} \frac{8 \pi \sqrt{2}}{h^{3}} m_{n}^{3 / 2} \frac{\left(1+\frac{2 E}{E_{g}}\right) \sqrt{E\left(1+\frac{E}{E_{g}}\right)}}{e^{\frac{E-\zeta_{n}}{k_{B} T}+1}} d E,
$$

where $E$ is energy, $k_{B}$ is the Boltzmann constant, and $h$ is the Planck's constant. The temperature dependencies of Fermi level position $\zeta_{p}$ relative to the top of the $T$-point band are calculated from

$$
p=\int_{-\infty}^{0} \frac{8 \pi \sqrt{2}}{h^{3}} m_{p}^{3 / 2} \frac{\sqrt{-E}}{e^{\frac{-E-\zeta_{p}}{k_{B} T}+1}} d E,
$$

where $m_{p}$ is the effective mass of $T$-point holes. The temperature dependencies of Fermi level positions $\zeta_{n}, \zeta_{p}$ are given in Figs. 6 and 7.

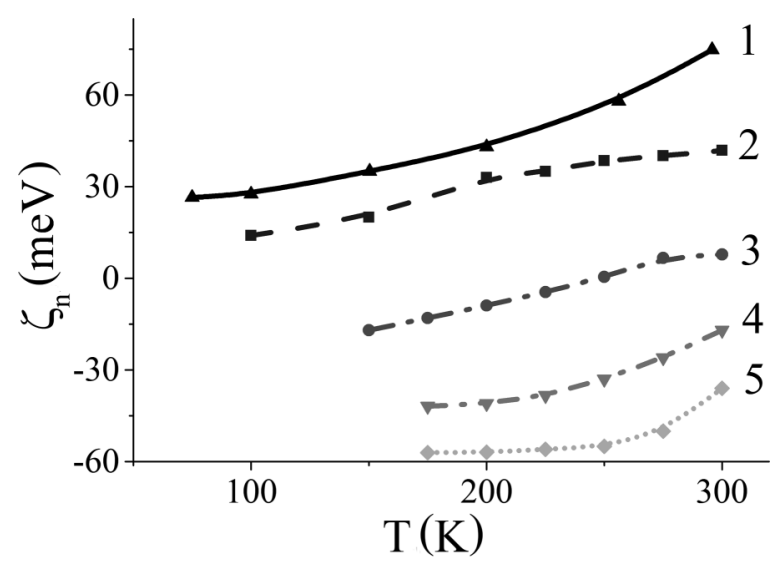

FIG. 7. Temperature dependencies of Fermi level position $\zeta_{n}$ relative to the bottom of band in the $L$ point for different $\mathrm{Sn}$ concentration $x: 1$ (0) (pure Bi), 2 (0.01 at.\%), 3 (0.02 at.\%), 4 (0.04 at.\%), $5(0.06$ at. \%). 


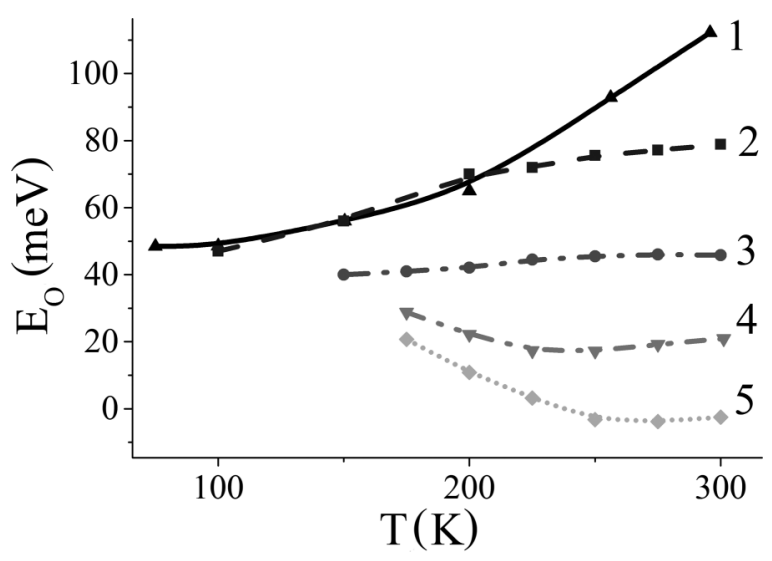

FIG. 8. Temperature dependence of $T$ - and $L$-point bands' overlap $E_{o}$ for different $\mathrm{Sn}$ concentration $x: 1(0)$ (pure Bi), 2 (0.01 at.\%), 3 (0.02 at.\%), 4 (0.04 at.\%), 5 (0.06 at.\%).

The doping shifts the Fermi level deeper inside the $T$-point band which leads to the increase of $p$ (Fig. 5). However, the increase of the temperature leads to the rollback and $\zeta_{p}$ tends to return closer to the original values in nondoped Bi (Fig. 6), reducing the $p$ with growth of temperature.

The comparison between estimated $\zeta_{p}$ and values [16,27] for single crystals found from Shubnikov-de Haas oscillations at LHT is given in the inset on Fig. 6. Our results (black squares) show quite good coincidence with the literature (white triangles).

\section{DISCUSSION}

\section{A. Temperature dependence of bands' overlap}

The Sn doping significantly shifts the Fermi level down relative to the bottom of the $L$-point electron band $\zeta_{n}$ in all the temperature ranges. The effect on the $\zeta_{p}$ is different: the change of Fermi level position is significant at low temperatures and is almost negligible at RT. This difference in outcome in temperature dependence of bands' overlap $E_{o}=\zeta_{n}+\zeta_{p}$ depends on doping level $x$ (Fig. 8).

The overlap $E_{o}$ between bands becomes smaller as $x$ increases (Fig. 8). Because the presence of small band overlap is often considered as a main feature of semimetals, it can be said that lessening of band overlap with $x$ turning from semimetal closer to narrow-gap semiconductor. This is illustrated by Fig. 9 for room temperature.

\section{B. Sommerfeld expansion prediction for Fermi level temperature dependence}

Sommerfeld expansion is the method of evaluating integrals of $\int_{-\infty}^{\infty} H(E) f(E) d E$ type, where $f(E)$ is Fermi distribution function. It is frequently being used for the calculation of temperature dependencies of fermion ensemble characteristics, e.g., Fermi level position. We have derived the expression and check if it is applicable to the $\mathrm{Bi}_{100-x} \mathrm{Sn}_{x}$ system Fermi level temperature dependencies.

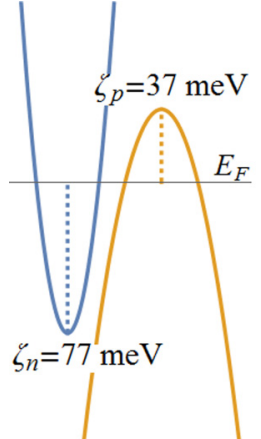

(a)

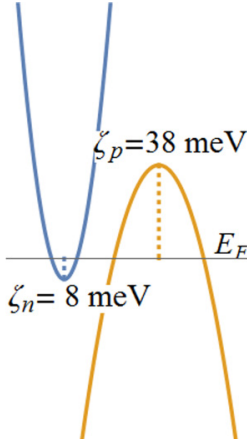

(b)

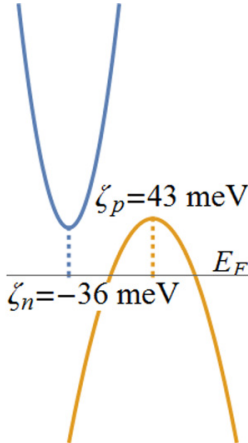

(c)
FIG. 9. The band structure of $\mathrm{Bi}_{100-x} \mathrm{Sn}_{x}$ at temperature $T=$ $300 \mathrm{~K}$ for (a) pure Bi, (b) $x=0.02$ at.\%, (c) $x=0.06$ at. $\%$. $E_{F}$ is Fermi level, $\zeta_{n}$ and $\zeta_{p}$ are the Fermi level positions relative to the band extrema in $L$ or $T$ points, respectively.

As follows from the Ashcroft and Mermin book [42], the integral $\int_{-\infty}^{\infty} H(E) f(E) d E$ can be reexpressed as

$$
\begin{aligned}
& \int_{-\infty}^{\infty} H(E) f(E) d E=\int_{-\infty}^{\zeta} H(E) d E \\
& \quad+\left.\sum_{n=1}^{\infty} \int_{-\infty}^{\infty} \frac{(E-\zeta)^{2 n}}{(2 n) !}\left(-\frac{\partial f}{\partial E}\right) d E \frac{d^{2 n-1}}{d E^{2 n-1}} H(E)\right|_{E=\zeta},
\end{aligned}
$$

where $\zeta$ is Fermi level, $H(E)$ some energy-dependent smooth function. By substituting the density of states $g(E)$ as $H(E)$ in Eq. (3) and keeping the first two temperature-dependent terms we get [42]

$$
\begin{aligned}
& \int_{-\infty}^{\infty} g(E) f(E) d E=\int_{-\infty}^{\zeta} g(E) d E \\
& +\left.\left[\int_{-\infty}^{\infty} \frac{(E-\zeta)^{2}}{2 !}\left(-\frac{\partial f}{\partial E}\right) d E \frac{d}{d E} g(E)\right]\right|_{E=\zeta} \\
& +\left.\left[\int_{-\infty}^{\infty} \frac{(E-\zeta)^{4}}{4 !}\left(-\frac{\partial f}{\partial E}\right) d E \frac{d^{3}}{d E^{3}} g(E)\right]\right|_{E=\zeta}+\cdots
\end{aligned}
$$

The first term can be rewritten in the assumption that the Fermi level change is small: $\int_{-\infty}^{\zeta} g(E) d E \approx \int_{-\infty}^{\zeta_{0}} g(E) d E+$ $\left(\zeta-\zeta_{0}\right) g(E)$, where $\zeta_{0}$ is the preknown Fermi level at low temperature. If the change in charge carrier concentration is small $\int_{-\infty}^{\infty} g(E) f(E) d E \approx \int_{-\infty}^{\zeta_{0}} g(E) f(E) d E$. That gives in our case

$\zeta(T)=\zeta_{0}-\frac{\pi^{2}}{6}\left(k_{B} T\right)^{2} \frac{g^{\prime}\left(\zeta_{0}\right)}{g\left(\zeta_{0}\right)}-\frac{7 \pi^{4}}{360}\left(k_{B} T\right)^{4} \frac{g^{\prime \prime \prime}\left(\zeta_{0}\right)}{g\left(\zeta_{0}\right)}-\cdots$.

The temperature dependence of the Fermi level position can be evaluated from Eq. (5) by substituting the expression for density of states (parabolic for holes or elliptical for electrons). The detailed derivation of expressions in case of elliptic density 


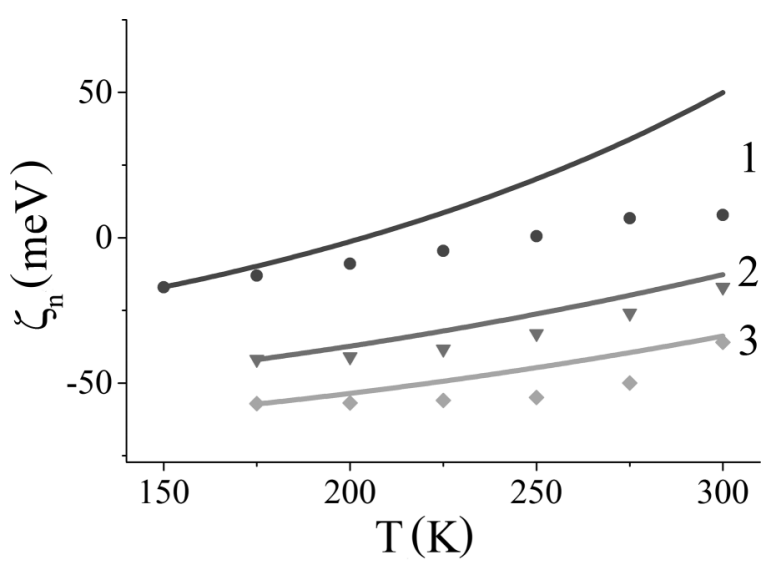

FIG. 10. Temperature dependence of Fermi level position $\zeta_{n}$ (scatter) and Sommerfeld expansion prediction (solid lines) for appropriate Sn concentration $x$ : 1 (0.02), 2 (0.04), 3 (0.06).

of states is given in Appendix F. We present the best coinciding with QMSA results in Figs. 10 and 11.

As seen from Fig. 10, $\zeta_{n}$ position predicted by Sommerfeld expansion shows in general the same tendency as QMSA data. It still keeps the monotonicity and does not deviate too much in absolute values for Sn concentrations $x>0.04$ (Fig. 10). In case of $\zeta_{p}$, the Sommerfeld expansion satisfactorily describes QMSA results only in the initial part of the temperature dependencies. For both $\zeta_{n}, \zeta_{p}$ the description by the Sommerfeld expansion better describes QMSA results with increase of $x$ (Fig. 11).

The results above show that Sommerfeld expansion is still limitedly applicable to the semimetallic systems. The limitation is connected to the assumption that carrier concentration changes slightly with temperature. The expansion better describes $\zeta_{n}$ with growth of $x$ because absolute values of electronic concentration $n$ decrease (Fig. 4), i.e., $n$ temperature changes become smaller as well. Only the initial part of $\zeta_{p}$ is well described because hole concentrations $p$ stay almost constant at low temperatures (Fig. 5).

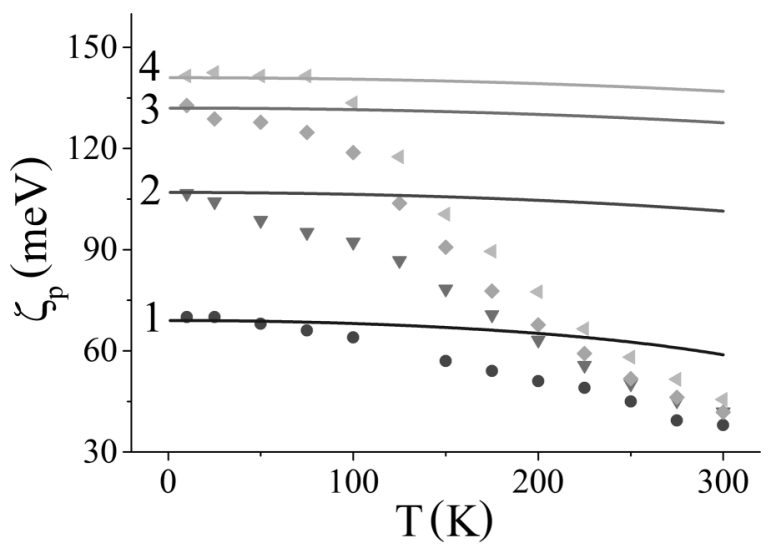

FIG. 11. Temperature dependence of Fermi level position $\zeta_{p}$ (scatter) and Sommerfeld expansion prediction (solid lines) for appropriate Sn concentration $x$ : 1 (0.02), 2 (0.04), 3 (0.06), 4 (0.08).

\section{CONCLUSIONS}

In summary, we have revealed three key features of band structure changes caused by temperature and doping in $\mathrm{Bi}_{100-x} \mathrm{Sn}_{x}(0 \geqslant x \geqslant 0.08$ at.\%) diluted alloys using the combination of QMSA, isotropic approximation for band structure, and Sommerfeld expansion method. This allowed to track Fermi level in wider temperature range (10-300 K) than was previously available in literature.

First, we have quantitatively established that band overlap $E_{o}$ decreases with the increase of Sn concentration. Second, it is demonstrated that the band overlap $E_{o}$ for samples with Sn concentration $x \geqslant 0.02$ at.\% reduces with temperature (reaching almost zero overlap for $x=0.08$ at. $\%, T=300 \mathrm{~K}$ ). These changes in electronic band structure may be interpreted as transition of $\mathrm{Bi}_{100-x} \mathrm{Sn}_{x}$ from semimetal to the narrow-gap semiconductor. The last, but not least, important result is that the Fermi level temperature dependencies can be qualitatively described by Sommerfeld expansion at low temperatures for samples with $x \geqslant 0.02$ at.\%.

\section{ACKNOWLEDGMENTS}

The authors gratefully acknowledge financial support by the Belarusian Republican Foundation for Fundamental Research in the framework of Project No. F16M-067 "Structure and magnetoresistive characteristics of $\mathrm{Bi}$ thin films," as well as by the German Academic Exchange Service (Deutscher Akademischer Austauschdienst, Grant No. 57214227). We thank our colleagues A. K. Fedotov and A. Mazanik from Belarusian State University who provided insight and expertise that greatly assisted the research. We thank S. Gusakova and S. Perevoznikov for the assistance with structure analysis.

\section{APPENDIX A: FABRICATION AND STRUCTURE OF SAMPLES}

\section{Fabrication}

The initial compounds were certified Bi and Sn of $99.9999 \%$ purity. The alloys were prepared in quartz tubes on air at temperatures 910-950 K. After crystallization, only the middle part of the obtained ingots were taken to the fabrication of foils. The stoichiometric proportion of compounds corresponded to $x=0.01,0.02,0.04,0.06,0.08$ at.\% in $\mathrm{Bi}_{100-x} \mathrm{Sn}_{x}$.

The electric motor rotated a copper cylinder of $20-\mathrm{cm}$ diameter with frequency $25 \mathrm{~Hz}$. The overheated melt was spilled out to the inner surface of cylinder . The alloy crystallized in stripes with length up to $10 \mathrm{~cm}$, width higher than $1 \mathrm{~cm}$, and thickness 20-60 $\mu \mathrm{m}$.

The speed $v$ of liquid phase cooling was evaluated [38] with Eq. (A1):

$$
v=\frac{\alpha \theta}{c d \rho}
$$

where $\alpha$ is the heat transfer coefficient, $\theta$ is the excess temperature of melt, $c$ is the heat capacity, and $d$ is the foil thickness. $\alpha$ lies in range $(1-2) \times 10^{5} \mathrm{~W} / \mathrm{m}^{2} \mathrm{~K}$, the difference in media and melt temperature was about $400 \mathrm{~K}$. All the other 


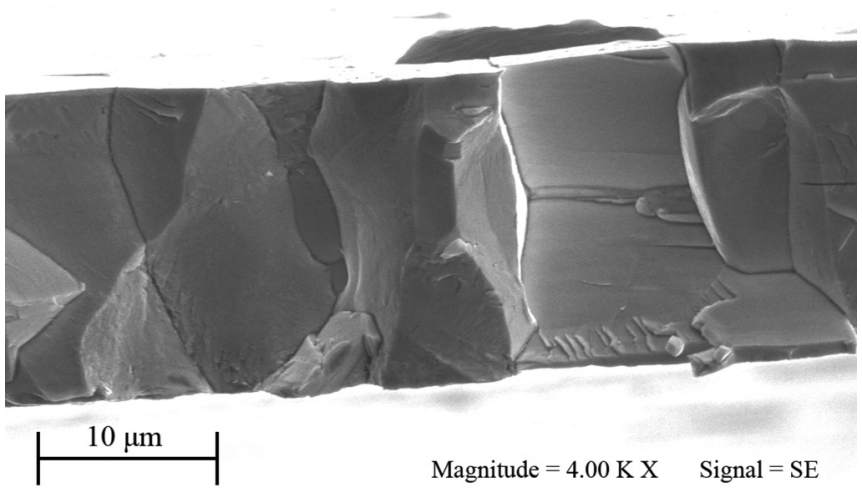

FIG. 12. Typical SEM image of $\mathrm{Bi}_{100-x} \mathrm{Sn}_{x}$ film cross section in secondary electron regime.

parameters were taken as for pure $\mathrm{Bi}$. Therefore, estimated cooling speed was $10^{6}-10^{7}\left(\mathrm{~K} \mathrm{~s}^{-1}\right.$ as in work [33].

For all further experiments, samples were cut from the center of the stripes to increase their homogeneity.

\section{Structure}

The cross sections of samples were studied using SEM in secondary electrons regime with LEO 1455VP Oxford Instruments microscope. Most grains in samples had rodlike shape (Fig. 12) due to high speed of cooling at synthesis.

Thickness of samples was estimated from scanning electron microscope (SEM) images of cross sections in secondary electrons regime and was $28-31 \mu \mathrm{m}$. The few percent error was introduced by image perspective distortion and nonsmoothness of chips. The crystalline texture and grain parameters were investigated with HKL Electron Backscatter Diffraction (EBSD) Premium System Channel 5 with 4QBSE Carl Zeiss detector or Bruker e-Flash HR EBSD Detector with an ARGUS FSE/BSE-Detector System.

The other details on grain structure are given in [33]. X-ray diffraction (XRD) patterns were measured with X-Pert PRO produced by PANalytical system (Netherland). The position of peaks was in agreement with electron backscatter diffraction (EBSD) (Fig. 13).

Crystalline texture was visualized in pole figures [Fig. 14(a)] and texture mapping in real space [Fig. 14(a)].

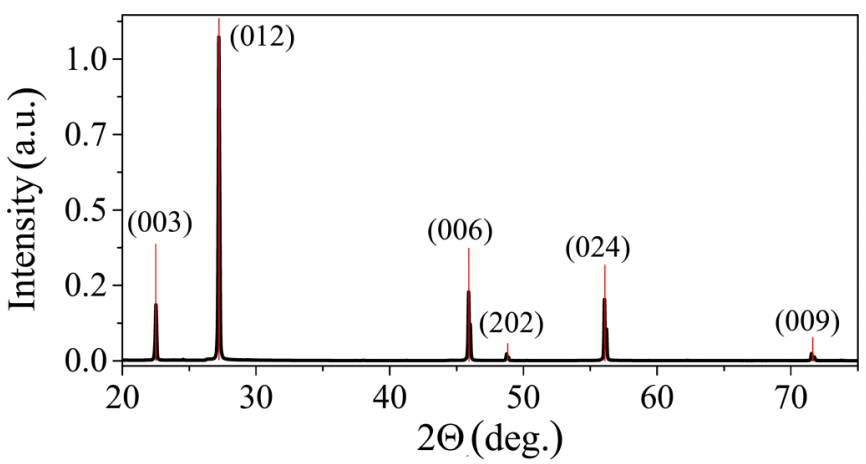

FIG. 13. Typical x-ray diffraction pattern of $\mathrm{Bi}_{100-x} \mathrm{Sn}_{x}$ sample.

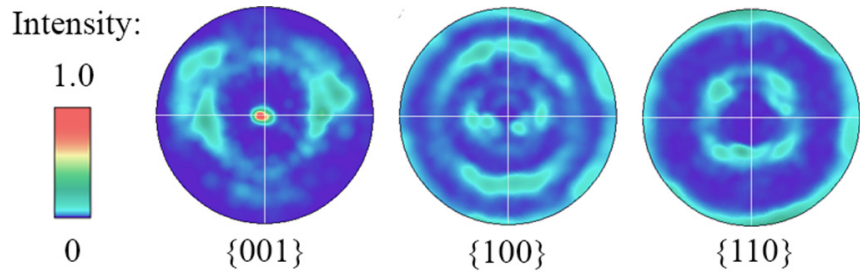

(a)

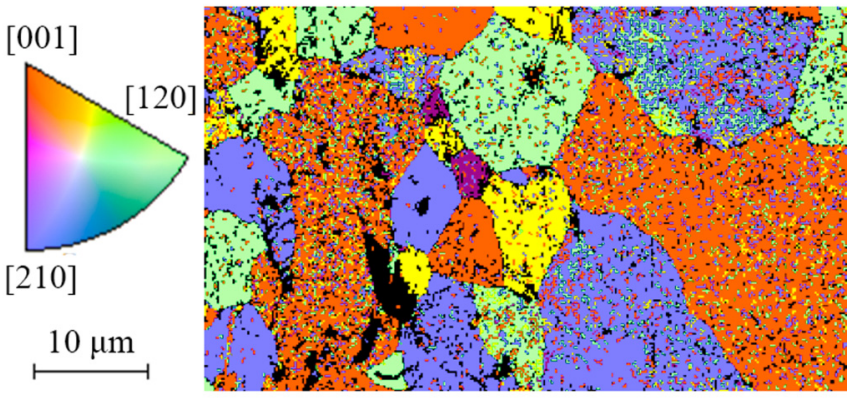

(b)

FIG. 14. Pole figures (a) and EBSD texture mapping (b) of samples. Indices are in hexahedral coordinate system.

Approximately $\frac{1}{3}$ of grains had orientation with direction [012] and $\frac{1}{3}$ with direction [001] in hexahedral coordinate system.

The relief of sample surface was investigated with atomic force microscopy (AFM) using an AutoProbe M5 setup produced by Park Scientific Instruments (Fig. 15). AFM showed that the deviations from mean thickness for each sample are less than $2 \%$.

The energy dispersive $\mathrm{x}$-ray measurements were performed with Ronteg EDX addon for SEM Leo 1455 VP. EDX analysis does not detect oversaturated with $\mathrm{Sn}$ regions in samples.

Element analysis was performed with PANalytical Epsilon $1 \mathrm{x}$-ray fluorescence spectrometer (XRF). Sn concentrations $x_{c h}$ detected by XRF and concentrations $x$ evaluated from the weight of initial compounds are given in Table I. As is seen from the table, detected $x_{c h}$ are smaller than by load. Taking in account the typical error of XRF measurements, we decided to point concentrations in the paper up to the one significant number.

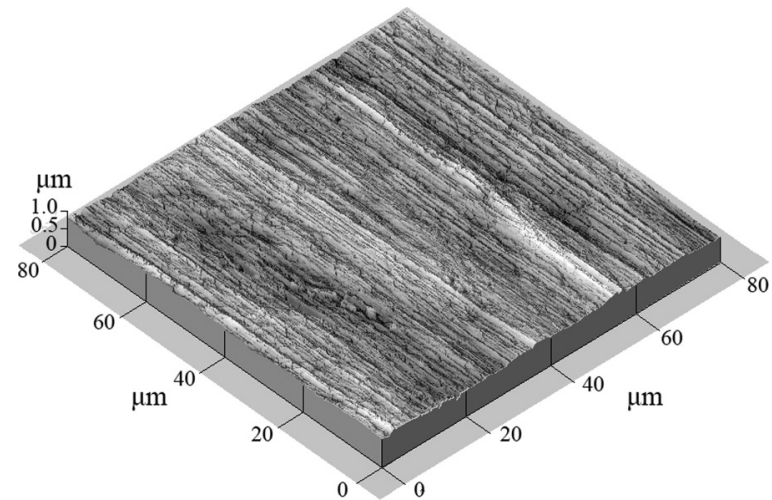

FIG. 15. Typical film surface image by AFM. 
TABLE I. Sn concentration $x$ evaluated by weight of initial compounds and Sn concentration $x_{c h}$ detected results of X-ray fluorescent analysis.

\begin{tabular}{llllll}
\hline \hline$x$ (at.\%) & 0.01 & 0.02 & 0.04 & 0.06 & 0.08 \\
$x_{\text {ch }}$ (at.\%) & 0.008 & 0.017 & 0.035 & 0.057 & 0.075 \\
\hline \hline
\end{tabular}

\section{APPENDIX B: GALVANOMAGNETIC MEASUREMENTS}

\section{Experiment details}

All measurements were performed on Cryogen Free Measurement System from Cryogenic Ltd. The covered range of temperatures is $5-300 \mathrm{~K}$ under magnetic fields up to $8 \mathrm{~T}$.

The samples were cut rectangular (typically $3 \times 8 \mathrm{~mm}$ ) from the middle of crystallized stripe, far from the stripe edges. Length and width of each sample were measured with optical microscope MPB-2 using collimator ruler which gives 0.01-mm error. Thickness of each chip was estimated from SEM measurements (see Appendix A). More details on microscopies are given in Appendix A.

The sample was wired on the contact pad (Fig. 16). Indium probes were deposited with ultrasound soldering. Six contacts were made: two current and two potential for four-point probe resistivity measurements and two potential for the Hall voltage measurements (Fig. 17). The uncertainty of contacts size was about $0.2 \mathrm{~mm}$ which gives $\approx 2 \%-3 \%$ error.

The dc was set in the range $10^{-4}-10^{-3}$ A with a Keithley Sub-Femtoamp RemoteSourceMeter SMU Instrument 6430 giving $0.05 \%$ error or less. Voltages were measured with Keithley 2182A Nanovoltmeters (error $0.01 \%$ in corresponding range). The contact pad with sample was placed in a cell with thermometer and magnetic field sensors in $\mathrm{He}$ atmosphere under low pressure. The cell was inserted into a superconducting solenoid inside of a cryostat. Temperature was regulated by gas He flow outside and electrical heaters inside the cell. Temperature was measured and stabilized using LakeShore 331 measuring controller providing the temperature determination with precision up to $0.01 \mathrm{~K}$.

While measuring Hall voltage, it is necessary to account influence of collateral galvanomagnetic and thermomagnetic effects as well as due to Hall contacts (Fig. 17) may be not equipotential under zero magnetic field. The measured voltage between Hall contacts (Fig. 17) consists of the following contributions [40]:

$$
U_{H C}=U_{H}+U_{N}+U_{E}+U_{R L}+U_{I R} .
$$

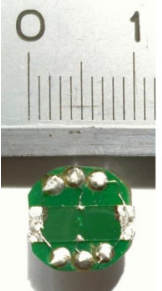

(a)

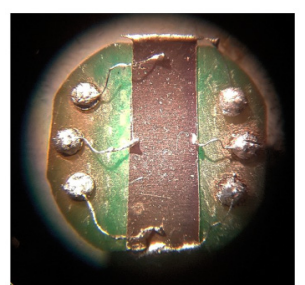

(b)
FIG. 16. The contact pad of galvanomagnetic probe (a) and the Bi sample mounted for the measurements (b).

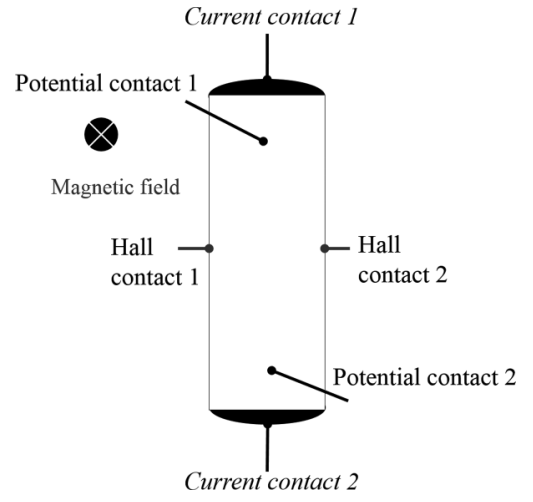

FIG. 17. Position of contacts on the sample.

Here, $U_{H}$ is voltage due to Hall effect, $U_{N}$ appears due to Nernst effect, $U_{E}$ and $U_{R L}$ are contributed by Ettingshausen and Righi-Leduc effects, $U_{I R}$ is the potential difference between Hall contacts in the absence of magnetic field. The sign of each contribution is defined by the sign of current and the magnetic field. There are four possible combinations of current and magnetic field directions:

$$
\begin{aligned}
& B+, I+: U_{H C}^{++}=U_{H}+U_{N}+U_{E}+U_{R L}+U_{I R}, \\
& B+, I-: U_{H C}^{+-}=-U_{H}+U_{N}-U_{E}+U_{R L}-U_{I R}, \\
& B-, I+: U_{H C}^{-+}=-U_{H}-U_{N}-U_{E}-U_{R L}+U_{I R}, \\
& B-, I+: U_{H C}^{--}=U_{H}-U_{N}+U_{E}-U_{R L}-U_{I R} .
\end{aligned}
$$

From Eqs. (B2)-(B5),

$$
U_{H}+U_{E}=\left(U_{H C}^{++}+U_{H C}^{+-}+U_{H C}^{-+}+U_{H C}^{--}\right) / 4 .
$$

Typically, $U_{H} \gg U_{E}$ so Hall voltage can be estimated with Eq. (B6).

The error in resistivity (and other specific characteristics) determination is mainly defined by the size of contacts and is equal or less than 5\%. Taking into account the inhomogeneity of current distribution, the overall error may achieve 5\%-7\%. The reproducibility of directly measured characteristics like potential differences is very good and the error is usually less than $1 \%$ according to our estimates.

\section{Primary data processing}

The result of a measurement is a data file with several columns: magnetic field induction on contact pad, temperature on sample, current through the sample $I$, potential difference in the direction of current flow and the Hall voltage. Automatized data processing is needed to build magnetic field dependencies of Hall coefficient $R_{H}(B)$, magnetoconductivity $\sigma_{x x}(B)$, and Hall conductivity $\sigma_{x y}(B)$.

The Hall coefficient $R_{H}$ is defined by Eq. (B7):

$$
R_{H}=d \frac{U_{H}}{I B} .
$$

Here, $d$ is sample thickness, $I$ is current, and $U_{H}$ is Hall voltage. The current direction was switched rapidly in each field point and mean Hall voltage was recorded. The magnetic field, in contrast, cannot be reversed quickly due to risk of 
additional heat release or even solenoid damage. Therefore, we measure monotonous dependence on field induction $B$ from 0 to $8 \mathrm{~T}, 8$ to $-8 \mathrm{~T}$, and back to zero. We interpolate measured $U_{H}(B)$ with first-order splines and set $U_{H}(B)$ as mean between two measurements $\left[U_{H}(B)-U_{H}(-B)\right] / 2$ for a number of $B_{j}$ field points. Then, a set of $R_{H}\left(B_{j}\right)$ values was calculated introducing averaged $U_{H}\left(B_{j}\right)$ both by field and current into Eq. (B7).

Relative magnetoresistance was estimated with Eq. (B8):

$$
\Delta \rho(B)=\rho(B)-\rho(0),
$$

where $\rho(B)$ is resistivity under field $B$.

Magnetoconductivity tensor components were computed [41] from resistivity under magnetic field $\rho(B)$ and Hall coefficient $R_{H}$ using the equations [41]

$$
\begin{aligned}
\sigma_{x x}(B) & =\frac{\rho(B)}{\rho(B)^{2}+R_{H}^{2} B^{2}}, \\
\sigma_{x y}(B) & =\frac{R_{H}(B) B}{\rho(B)^{2}+R_{H}^{2} B^{2}} .
\end{aligned}
$$

\section{APPENDIX C: ELECTRICAL PROPERTIES}

\section{Resistivity, magnetoresistance, Hall coefficient}

The temperature dependence of resistivity was measured in the range 10-300 K (Fig. 18). Resistivity of pure Bi 1 increases linearly with temperature. The addition of tin increases the resistivity. There are small extrema for doped samples 2-6 which are shifted to higher temperatures for more doped samples.

Hall coefficient $R_{H}$ was estimated in the temperature range 10-300 K under fields up to $8 \mathrm{~T}$ (Figs. 19 and 20). Hall coefficient $R_{H}$ of isotropic material with two carrier types [41] can be approximated at low fields as

$$
R_{H}=-\frac{r_{H}}{q}\left(\frac{n \mu_{n}^{2}-p \mu_{p}^{2}}{n^{2} \mu_{n}^{2}+p^{2} \mu_{p}^{2}}\right)
$$

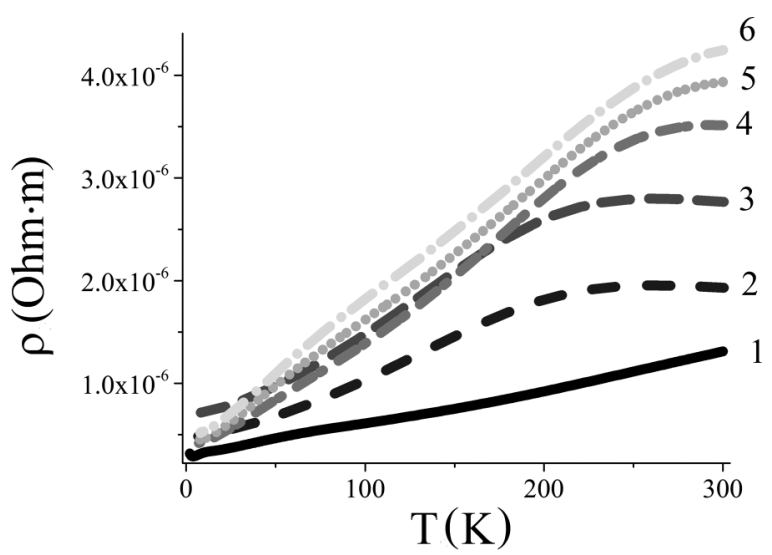

FIG. 18. Resistivity temperature dependence $\rho(T)$ for various concentrations $x$ (at.\%) of Sn: 1 (0) (pure Bi), 2 (0.01), 3 (0.02), 4 (0.04), 5 (0.06), 6 (0.08).

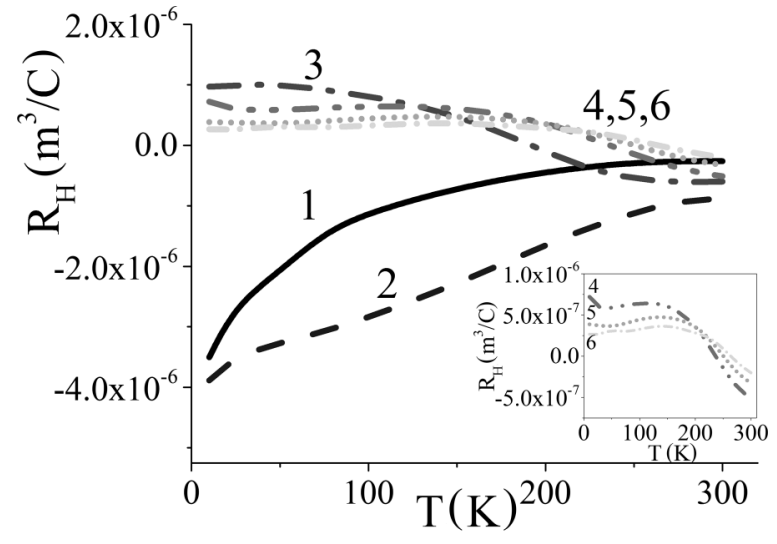

FIG. 19. Temperature dependence of Hall coefficient $R_{H}$ under field $B=0.1 \mathrm{~T}$ for various concentrations $x$ (at.\%): 1 (0) (pure Bi), 2 (0.01), 3 (0.02), 4 (0.04), 5 (0.06), 6 (0.08).

and at highs fields as

$$
R_{H}=\frac{r_{H}}{q}\left(\frac{1}{p-n}\right) .
$$

Here $p, n$ are concentrations, $\mu_{p}, \mu_{n}$ are the mobilities of holes and electrons, respectively, $q$ is the elementary charge, and $r_{H}$ is the Hall factor which depends on scattering mechanism and is close to 1 .

The sign of low-field Hall coefficient [Eq. (C1)] is governed by carriers with highest product of concentration $n$ by squared mobility $\mu^{2}$. For pure $\mathrm{Bi}$, the Hall coefficient is negative: mobility of electrons in $\mathrm{Bi}$ is significantly higher than one of holes, whereas concentrations are the same. Moreover, the lowfield Hall coefficient (Fig. 19) remains negative for all studied $x$ at room temperatures. It confirms that relative contribution of electrons substantially increases with temperature $\left[\mu_{n}^{2} n\right.$ term prevails according Eq. (C1)].

Hall coefficient in high-field limit [see Eq. (C2)] must be independent on mobilities. Hall coefficients of doped samples do not saturate in available fields so it is hard to make quantitative interpretations. However, we can still use $R_{H}$ at $8 \mathrm{~T}$ to obtain qualitative information about material. The $R_{H}$

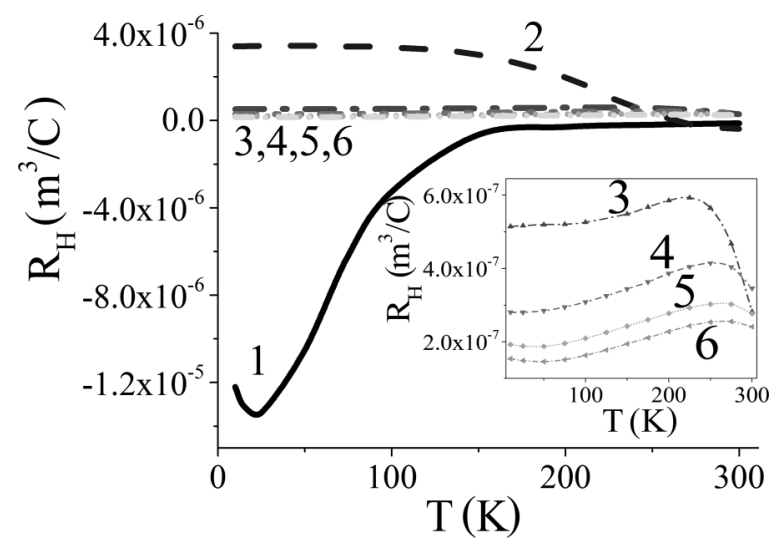

FIG. 20. Temperature dependence of Hall coefficient $R_{H}$ under field $B=8 \mathrm{~T}$ for various concentrations $x$ (at.\%): 1 (0) (pure $\mathrm{Bi}$ ), 2 (0.01), 3 (0.02), 4 (0.04), 5 (0.06), 6 (0.08). 
sign under $8 \mathrm{~T}$ becomes positive which marks that majority of carriers are holes (Fig. 20). Samples with $x \geqslant 0.02$ at.\% always show positive Hall coefficient at $8 \mathrm{~T}$. Although high-field limit is not achieved, $R_{H}$ at $8 \mathrm{~T}$ field are relatively independent on mobilities of charge carriers in comparison to the low-field case. Therefore, majority of charge carriers (by concentrations) are holes and amount of its excess increases with $x$ [follows from Eq. (C2), absolute values of $R_{H}$ at $8 \mathrm{~T}$ decreases].

It is curious to compare the signs of $R_{H}$ of doped samples at low (Fig. 19) and high fields (Fig. 20). Hall coefficients at low field become negative much earlier than $R_{H}$ at high field (see $R_{H}$ temperature dependencies). It means that although excess carriers are holes, the contribution of electrons to conduction at temperatures upper than $200 \mathrm{~K}$ is comparable to the one of holes (Fig. 19).

\section{Magnetoconductivity tensor components}

The $\sigma_{x x}$ field dependencies are given in Fig. 21. Field dependencies of $\sigma_{x x}$ show no peculiarities and are in agreement with resistivity/magnetoresistance temperature dependencies (Figs. 18 and 3). The relative MR of nondoped Bi and sample with $x=0.01$ at.\% are similar (see Fig. 18), while the absolute values of resistivity are lower by an order of magnitude. Theoretically, it may open the possibility for fabrication of magnetoresistors with controllable absolute resistivity and relative $\mathrm{MR}$ as high as of $\mathrm{Bi}$ at room temperatures.

The $\sigma_{x y}$ data are given on Fig. 22. Field dependencies of $\sigma_{x y}$ are valuable for qualitative analysis of mobilities: the field $B$ where $\sigma_{x y}$ shows extrema is proportional to inverse mobility of charge carriers (maximum corresponds to holes and minimum to electrons). Therefore, the lower field where $\sigma_{x y}$ shows extrema means higher mobility of charge carriers.

At low temperatures, the mobility of electrons in pure $\mathrm{Bi}$ is higher than inverse lowest available fields in our setup. Therefore, we see only the right side of the minimum [Figs. 22 (a) and 22(b)].

Addition of tin shifts the minimum to higher fields which means reduction of electron mobility. Maxima become distinguishable since $x \geqslant 0.01 \%$ and appear under higher fields than minima (Fig. 22), which means lower mobility than one of electron. For $x \geqslant 0.04 \%$, the hole peak exhibits significant broadening at lower temperatures which may be considered as contribution of the second, high-mobility hole species.

The general trend is that mobility of charge carriers decreases with doping level and temperature. Mobility of electrons is higher than one of holes for all the studied cases. Electron contribution to conductivity is seen for all the samples at room temperatures as can be seen from sign of $\sigma_{x y}$.

\section{Why QMSA is required in this particular research}

The QMSA is a complicated numerical procedure and may not be familiar even to many of the specialists in semiconductor science. Therefore, we feel obligated to justify our choice of this tool.

It is well known that mobility of charge carrier can be estimated as $B_{i}^{-1}$ where $B_{i}$ is field and where $\sigma_{x y}(B)$ shows the corresponding peak. But, in case that there several types of charge carriers with comparable mobilities, the related peaks

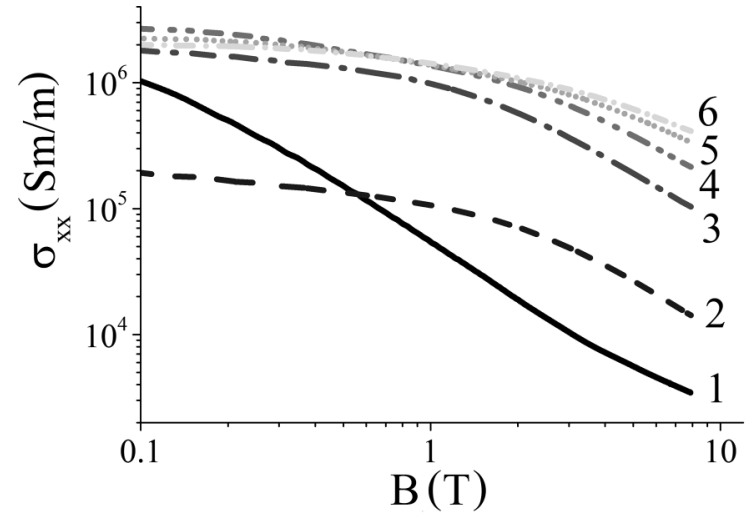

(a)

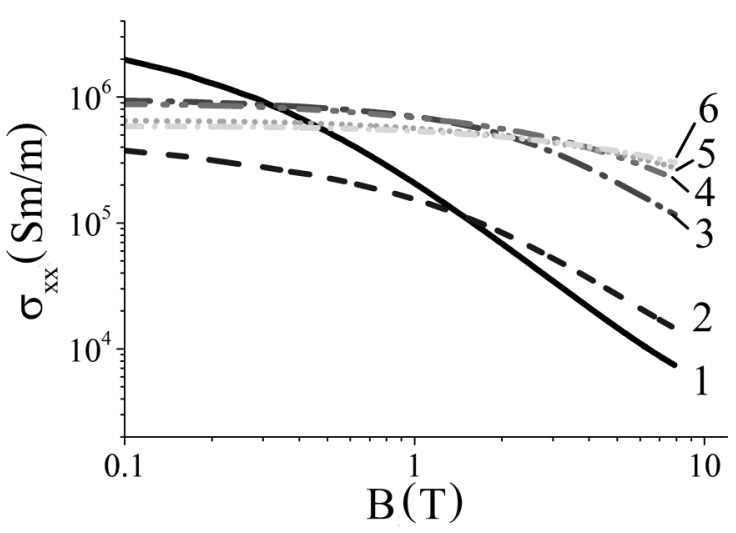

(b)

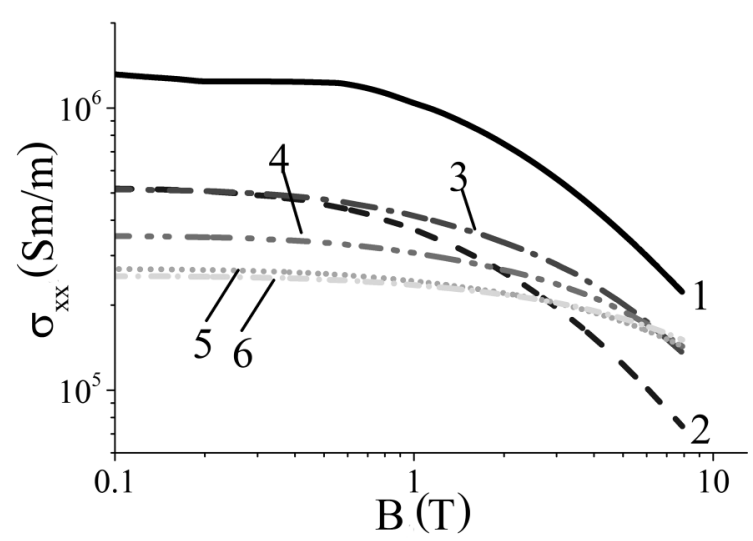

(c)

FIG. 21. Dependence of $\sigma_{x x}$ on magnetic field $B$ at temperatures (a) $10 \mathrm{~K}$, (b) $100 \mathrm{~K}$, (c) $300 \mathrm{~K}$ for different concentrations $x$ (at. $\%$ ): 1 (0) (pure Bi), 2 (0.01), 3 (0.02), 4 (0.04), 5 (0.06), 6 (0.08).

will distort each other. Let us illustrate this on the basis of our samples.

The temperature dependencies of $T$-point hole mobilities $\mu_{p L}$ calculated from the inverse position of $\sigma_{x y}(B)$ peaks are given on Fig. 23. For nondoped $\mathrm{Bi}, \sigma_{x y}$ is negative at all $B$ and holes cannot be seen on $\sigma_{x y}(B)$ dependencies directly.

On Fig. 23, the mobility of sample (2) with $x=0.01 \%$ rises with temperature at $10-100 \mathrm{~K}$. There is no sense in huge decrease of (2) $\mu_{p L}$ at $T>150$ because it makes mobilities for 


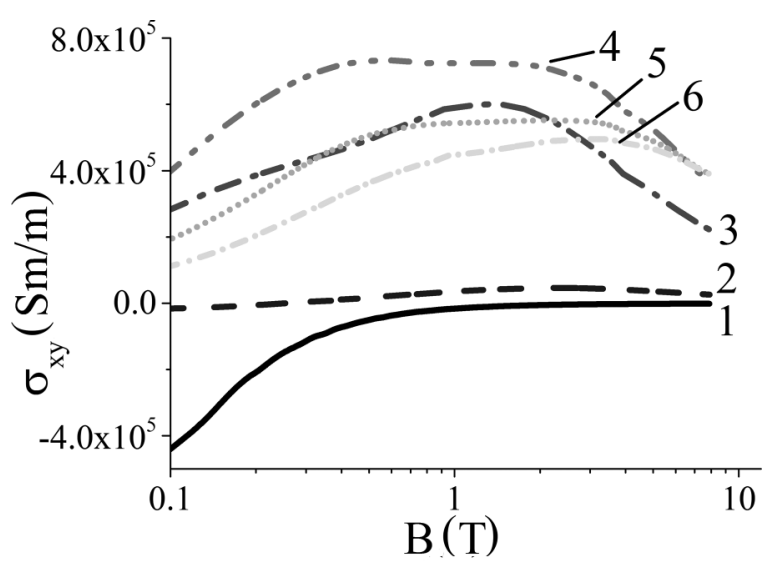

(a)

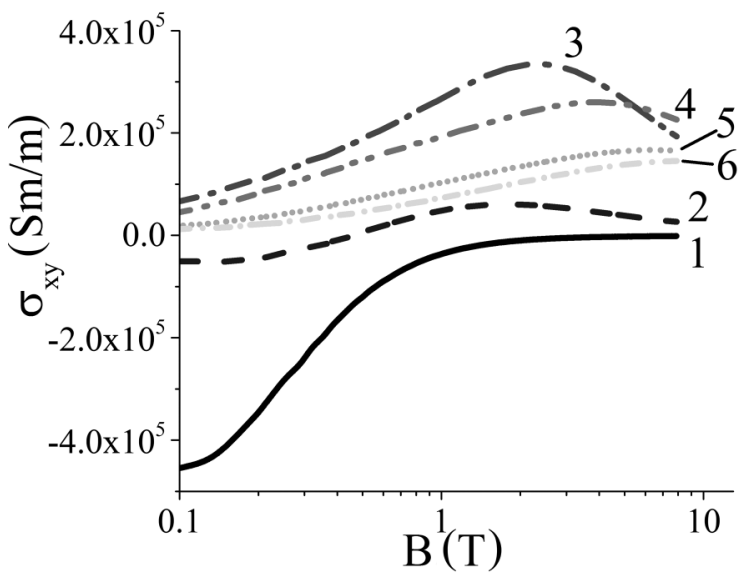

(b)

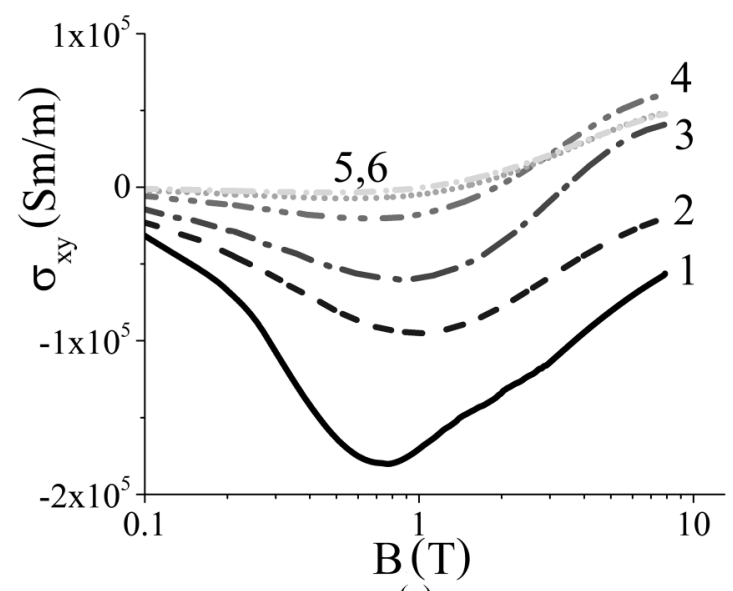

(c)

FIG. 22. Dependence of $\sigma_{x y}$ on magnetic field $B$ at temperatures (a) $10 \mathrm{~K}$, (b) $100 \mathrm{~K}$, (c) $300 \mathrm{~K}$ for different concentrations $x$ (at. \%): 1 (0) (pure Bi, not seen), 2 (0.01), 3 (0.02), 4 (0.04), 5 (0.06), 6 (0.08).

sample $x=0.01$ at. $\%$ even lower than hole mobilities in more doped samples. The last is an artifact caused by the electrons which influence on $\sigma_{x y}(B)$ cancels the neighboring part of positive peak related to holes. The hole mobilities temperature dependence for rest $x \geqslant 0.02 \%$ seems more or less realistic.

The situation with electrons is not so good. According to Fig. 24, electron mobility rises several times with increase of dopant concentration. Truly, a small rise is possible due to

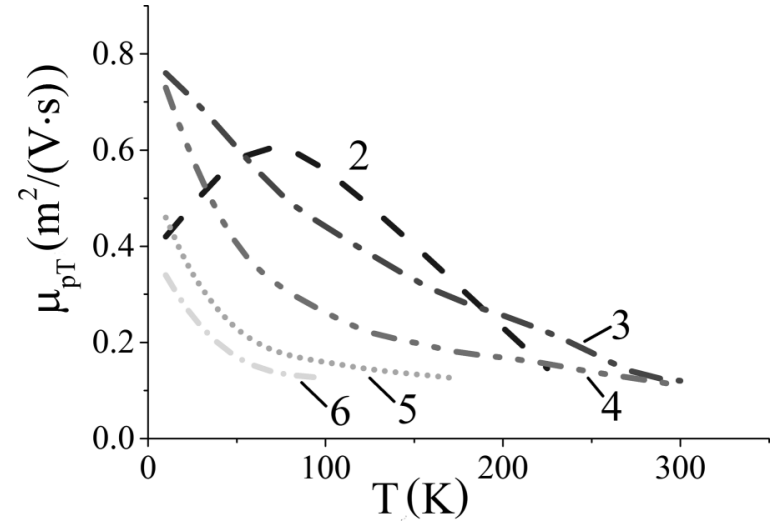

FIG. 23. Mobility of $T$-point holes calculated directly from position of $\sigma_{x y}$ extrema $x$ (at.\%): 1 (0) (pure Bi), $2(0.01), 3(0.02), 4(0.04)$, 5 (0.06), 6 (0.08) (not seen).

narrowing of the conduction band in the $L$ point, but such a serious rise is wrong for sure. The reasons for this serious distortion of negative parts of $\sigma_{x y}$ field dependencies (Fig. 22) is caused by holes. In samples with low influence of holes ( $x=0 \%, 0.01 \%$ ), electron mobilities look realistic.

The QMSA methods were developed to take into account the mutual influence of different charge carriers to corresponding regions of $\sigma_{x y}$ field dependence. This is why we decided to use it in our study.

\section{APPENDIX D: QUANTITATIVE MOBILITY SPECTRUM ANALYSIS IMPLEMENTATION}

Quantitative mobility spectrum analysis (QMSA) is a common name for the modern family of techniques for processing data from galvanomagnetic measurements. Great advantage of QMSA is the possibility of extraction of charge carrier mobilities and concentrations in cases where traditional equations for kinetic coefficients give significant error or require too many additional data. The main fields of QMSA application are materials with several sorts of charge carriers $[11,36,43]$ ). Numerical implementations of QMSA have gone a long way in a few decades. In the pioneering approach by Beck and

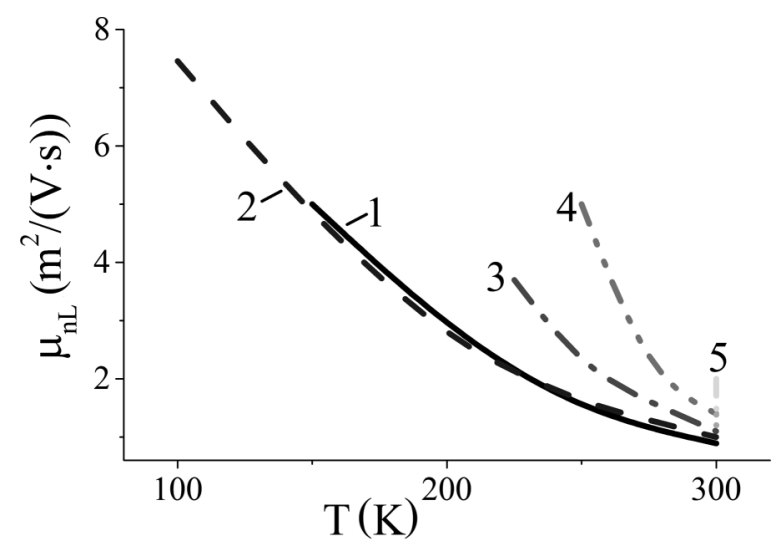

FIG. 24. Mobility of $L$-point electrons calculated directly from position of $\sigma_{x y}$ extrema $x$ (at.\%): 1 (0) (pure Bi), 2 (0.01), 3 (0.02), 4 (0.04), 5 (0.06), 6 (0.08). 
Anderson [43], the condition for uniqueness was that the solution is the envelope rather than the true mobility spectrum and is inaccurate [36]. Modern algorithms are much more accurate and uniqueness of fit in correctly set problems is mentioned in several reports $[36,44]$.

The underlying physics of QMSA is usually $[10,34,35,37,43,44]$ based on single charge carrier McClures [45] solution for magnetoconductivity tensor components $\sigma_{x x}$, $\sigma_{x y}$ in magnetic field $B$ :

$$
\begin{aligned}
& \sigma_{x x}(B)=\frac{q n\langle\mu\rangle}{1+\langle\mu\rangle^{2} B^{2}}, \\
& \sigma_{x y}(B)=\frac{q n\langle\mu\rangle^{2} B}{1+\langle\mu\rangle^{2} B^{2}},
\end{aligned}
$$

where $q,\langle\mu\rangle$, and $n$ are carrier's charge, mean mobility, and concentration.

To get the form suitable for QMSA procedure, we need to account distributions of carrier mobility vs carrier energy instead of single $\langle\mu\rangle$. This can be done in two ways: one [34] is to present $n$ as a function of mobility $n(\mu)$ and integrate $\frac{\partial n(\mu)}{\partial \mu} d \mu$, the next is to replace $\langle\mu\rangle$ by summation over a set of delta functions $\mu_{i}[10,44]$. We choose the last way because it is both more developed and simpler to implement. Equations (D1) and (D2) become [46]

$$
\begin{gathered}
\sigma_{x x}(B)=\sum_{i=1}^{M} \frac{q_{i} n_{i} \mu_{i}}{1+\mu_{i}^{2} B^{2}}, \\
\sigma_{x y}(B)=\sum_{i=1}^{M} \frac{q_{i} n_{i} \mu_{i}^{2} B}{1+\mu_{i}^{2} B^{2}} .
\end{gathered}
$$

Here, $M$ is number $i$ of points (delta functions) in mobility mesh.

It is also convenient to unite $q_{i} n_{i} \mu_{i}$ in Eqs. (D3) and (D4) numerators to introduce partial conductivities:

$$
\begin{aligned}
\sigma_{x x}\left(B_{j}\right) & =\sum_{i=1}^{M} \frac{\sigma_{p}\left(\mu_{i}\right)+\sigma_{n}\left(\mu_{i}\right)}{1+\mu_{i}^{2} B_{j}^{2}}, \\
\sigma_{x y}\left(B_{j}\right) & =\sum_{i=1}^{M} \frac{\left[\sigma_{p}\left(\mu_{i}\right)-\sigma_{n}\left(\mu_{i}\right)\right] \mu_{i} B_{j}}{1+\mu_{i}^{2} B_{j}^{2}} .
\end{aligned}
$$

Here, partial hole and electron contributions are $\sigma_{p}\left(\mu_{i}\right)=$ $|q| p_{i} \mu_{i}$ and $\sigma_{n}\left(\mu_{i}\right)=|q| n_{i} \mu_{i}, q$ is elementary charge. The main goal is to find the distributions of $\sigma_{p}$ and $\sigma_{n}$ vs mobility. The technical core of QMSA is nonlinear least-square fitting. The short description comes below.

First, we fill the array of $\sigma_{p}(\mu)$ and $\sigma_{n}(\mu)$ with initial positive values. Then, we calculate $\sigma_{x x}^{\text {trial }}$ and $\sigma_{x y}^{\text {trial }}$ by Eqs. (D5) and (D6) from $\sigma_{p}(\mu)$ and $\sigma_{n}(\mu)$ under several field $B$ values. The key moment of least-square methods is the estimation of total squared error. Vurgaftman and others suggested [44] to include derivatives and proved that it leads to smoother spectra:

$\chi^{2}=\sum_{j=1}^{N} \frac{1}{N} \frac{\left(\Delta \sigma_{x x, j}\right)^{2}+\left(\Delta \sigma_{x y, j}\right)^{2}+\left(\Delta \sigma_{x x, j}^{\prime}\right)^{2}+\left(\Delta \sigma_{x y, j}^{\prime}\right)^{2}}{\left(\sigma_{x x, j}^{\exp }\right)^{2}+\left(\sigma_{x y, j}^{\exp }\right)^{2}+\left(\left[\sigma_{x x, j}^{\exp }\right]^{\prime}\right)^{2}+\left(\left[\sigma_{x y, j}^{\exp }\right]^{\prime}\right)^{2}}$.
Here, $N$ is the number of available points in magnetic field, $\sigma_{x x, j}^{\exp }$ and $\sigma_{x y, j}^{\exp }$ are the experimentally measured magnetoconductivity tensor components, and $\sigma_{x x, j}^{\text {trial }}$ and $\sigma_{x y, j}^{\text {trial }}$ are the tensor components calculated by Eqs. (D5) and (D6) from $\sigma_{p}, \sigma_{n}$ vs $\mu$. In the numerator, $\Delta \sigma_{x x, j}=\sigma_{x x, j}^{\text {expt }}-\sigma_{x x, j}^{\text {trial }}$ and $\Delta \sigma_{x y, j}=$ $\sigma_{x y, j}^{\text {expt }}-\sigma_{x y, j}^{\text {trial }}$. The other terms includes field derivatives:

$$
\begin{aligned}
& {\left[\sigma_{x x, j}^{\text {expt }}\right]^{\prime}=\left.B_{j} \frac{\partial \sigma_{x x, j}^{\text {expt }}}{\partial B}\right|_{B=B_{j}},} \\
& {\left[\sigma_{x y, j}^{\text {expt }}\right]^{\prime}=\left.B_{j} \frac{\partial \sigma_{x y, j}^{\text {expt }}}{\partial B}\right|_{B=B_{j}} \cdot} \\
& \Delta \sigma_{x x, j}^{\prime}=\left.B_{j}\left(\frac{\partial \sigma_{x x, j}^{\text {expt }}}{\partial B}-\frac{\partial \sigma_{x x, j}^{\text {trial }}}{\partial B}\right)\right|_{B=B_{j}}, \\
& \Delta \sigma_{x y, j}^{\prime}=\left.B_{j}\left(\frac{\partial \sigma_{x y, j}^{\text {expt }}}{\partial B}-\frac{\partial \sigma_{x y, j}^{\text {trial }}}{\partial B}\right)\right|_{B=B_{j}} .
\end{aligned}
$$

The next step is decreasing the error by modification of $\sigma_{p}(\mu)$ and $\sigma_{n}(\mu)$. The approach described in improved QMSA [44] suggests to make small change $\sigma_{p / n}(\mu)$ only in a single point per iteration, but the change in this point must provide the higher decrease of error than possible small changes at any of the rest $\mu_{i}$ points.

The values of $\sigma_{p / n}\left(\mu_{i}\right)$ can be increased by multiplying $(1+f)$, decreased by $(1-f)$, where $f<1$ is adjustment factor [see Eq. (D12)], or stay the same as on previous iteration. Option where both $\sigma_{p / n}\left(\mu_{i}\right)$ stay not changed can be dropped because it means no modification at all. For rest $M \mu_{i}$ points there will be $(8 M)$ possible adjustments at one iteration. We calculate all corresponding new $(8 M)$ errors and sort them in order to find the smallest. After it is found, we change corresponding $\sigma_{p}\left(\mu_{i}\right)$ and $\sigma_{n}\left(\mu_{i}\right)$ and proceed to the next iteration

$$
\sigma_{\text {new }}\left(\mu_{i}\right)=(1 \pm f) \sigma_{\text {old }}\left(\mu_{i}\right) .
$$

We also implemented the fitting in Fourier space to accelerate convergence and decrease the number of artifacts [10]. It was very similar to the procedure described above. It is only needed to transfer in Fourier domain with discrete Fourier transforms applied to $\sigma_{p}\left(\mu_{i}\right)$ and $\sigma_{n}\left(\mu_{i}\right)$ arrays:

$$
\tilde{S}\left(\mu_{l}^{-1}\right)=\sum_{k=1}^{M} \sigma\left(\mu_{k}\right) e^{-\frac{2 \pi i}{M}(k-1)(l-1)},
$$

where $\tilde{S}\left(\mu_{i}^{-1}\right)$ is the Fourier image of corresponding $\sigma\left(\mu_{i}\right)$ spectrum. The adjustment of the image

$$
\tilde{S}_{\text {new }}\left(\mu_{l}^{-1}\right)=(1 \pm f) \cdot \tilde{S}_{\text {old }}\left(\mu_{l}^{-1}\right)
$$

and inverse transform is needed to calculate the error

$$
\sigma_{\text {new }}\left(\mu_{k}\right)=\frac{1}{M} \sum_{l=1}^{M} \tilde{S}_{\text {new }}\left(\mu_{l}^{-1}\right) e^{\frac{2 \pi i}{M}(k-1)(l-1)} .
$$

The uniqueness of mobility spectra was discussed in Beck and Anderson's pioneering article [43]. They showed that the envelope of mobility spectra is unique. This envelope literally 


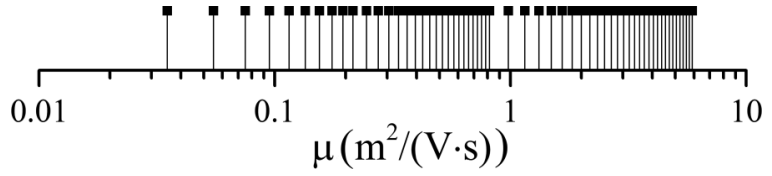

FIG. 25. Example of mobility mesh with two scales needed to accurately capture both carriers with higher and lower mobilities.

contains all meaningful information that can be extracted from galvanomagnetic measurements. Antoszewski and others discussed [36] convergence of improved QMSA. They analyzed the set of galvanomagnetic data specimen (both experimental and synthetic) and established that convergence has been obtained for every reasonable data set ever analyzed.

The initial conditions for the best convergence for similar algorithm were discussed [44] by Vurgaftman and others. In this early implementation, improved QMSA begins with an initial trial spectrum, which for example may be conveniently taken from the Beck and Anderson algorithm. It should be noted, they said, that the precise form of the initial trial spectrum has little influence on the final result. Therefore, improved QMSA seems stable relative to initial conditions.

As our algorithm implements the feature of Fourier-domain fitting, it is fair to apply arguments from the original paper [10] that introduces Fourier analysis to QMSA. Unlike prior methods requiring a separate computational algorithm for the envelope from Beck and Anderson as the initial guess, the FMSA method quickly gets a more accurate envelope after its first resolution step with lowest spectral resolution and least fitted data points. It starts with a constant carrier conductivity for all the mobility points. Therefore, Cui et al. report [10] that implementation of Fourier-domain fitting allows the usage of constant values as initial spectrum form.

The implementation was written in Intel Visual Fortran 2015. The example of mobility mesh is given in Fig. 25. The mesh was made nonuniform (denser at low mobility values) in order to guarantee needed the number of points for the good resolution of low-mobility holes' peak.

The code tested on synthetic data (see Fig. 26) consisted of one electron and one hole species with mobilities differing by an order. The forms of synthetic $\sigma_{p}\left(\mu_{i}\right), \sigma_{n}\left(\mu_{i}\right)$ distribution were taken Gaussian.

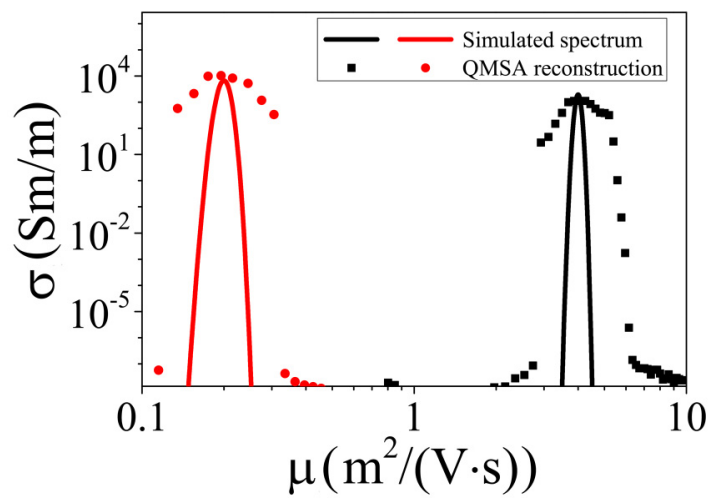

FIG. 26. Simulated (solid lines) and reconstructed (dots) mobility spectra.

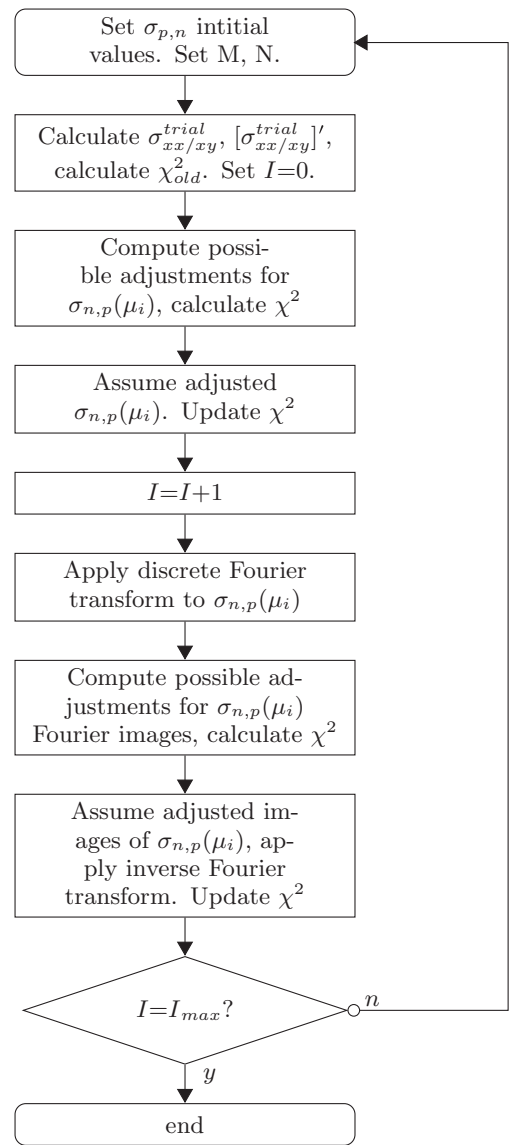

FIG. 27. Algorithm of QMSA implementation with Fourierdomain adjustment and derivatives taken into account for error calculation.

QMSA procedure run with adjustment factor of $f=0.05 \%$ and mesh with 60 points (see Fig. 27). 300 interpolated field points in the range $0.01-8 \mathrm{~T}$ were taken. The procedure was numerically heavy and took several hours for 12 data sets to complete with achievement 10 ${ }^{-1} \%$ error on Intel Xeon E5-2695.

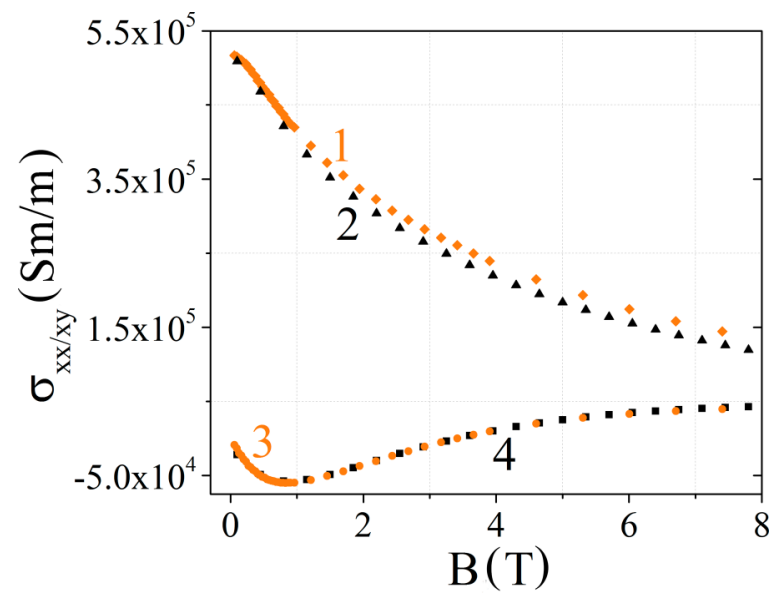

FIG. 28. Reconstructed $\sigma_{x x}$ (1, orange diamonds), $\sigma_{x y}$ (2, black triangles) and measured $\sigma_{x x}$ (3, orange circles), $\sigma_{x y}$ (4, black squares) for $\mathrm{Bi}_{0.9998} \mathrm{Sn}_{0.0002}$ at $T=300 \mathrm{~K}$. 
The result of simulated data reconstruction was generally correct with a small broadening (see Fig. 26), which is common for the QMSA family of methods.

The example of real data fit by the described algorithm is given in Fig. 28. The black points are experimental data and orange points are evaluated from mobility spectra by Eqs. (D1)

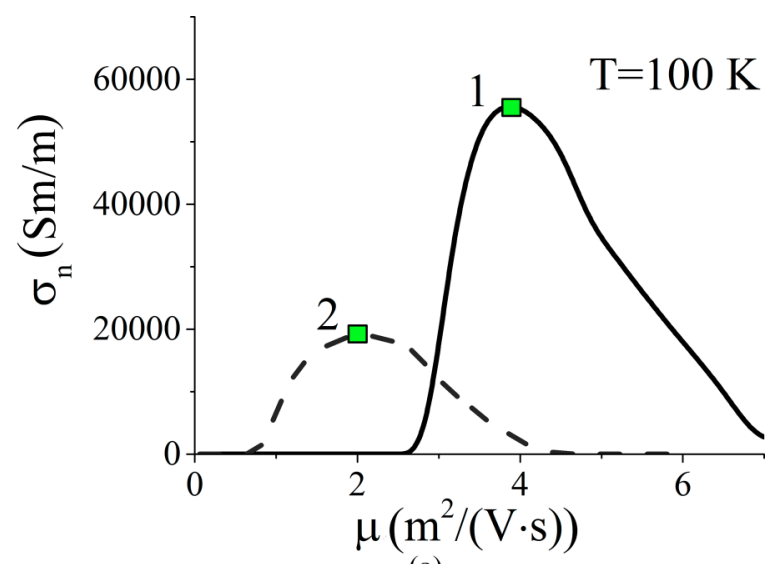

(a)

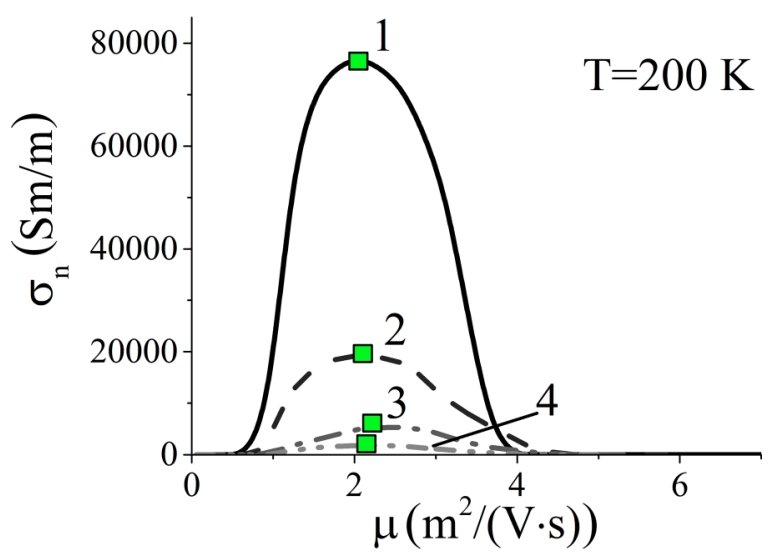

(b)

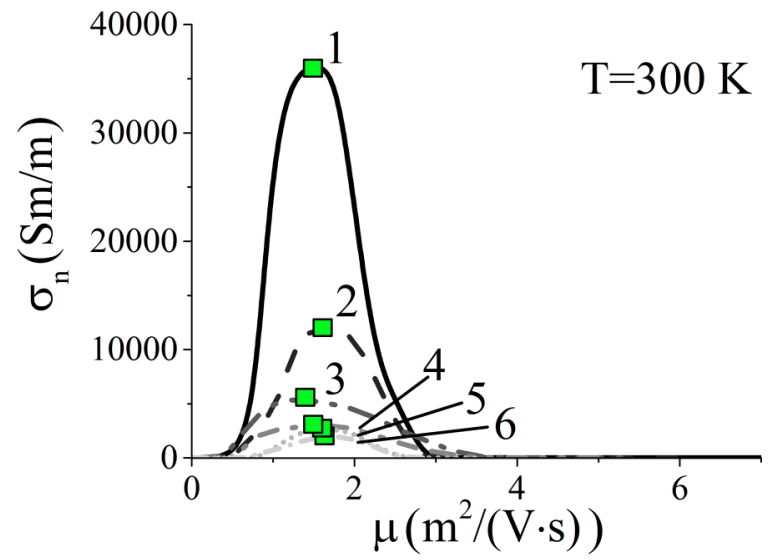

(c)

FIG. 29. Distribution of electron partial conductivity (main peak) $\sigma_{n}(\mu)$ of $\mathrm{Bi}_{100-x} \mathrm{Sn}_{x}$ vs mobility $\mu$ at different temperatures: $100 \mathrm{~K}$ (a), $200 \mathrm{~K}$ (b), $300 \mathrm{~K}$ (c). Number marks $x$ (at.\%) of Sn: 1 (0) (pure $\mathrm{Bi}), 2$ (0.01), 3 (0.02), 4 (0.04), 5 (0.06), 6 (0.08). Green squares mark centers of peaks. and (D2). The coincidence of measured and reconstructed data is good and in agreement with low $\chi^{2}$ error.

\section{APPENDIX E: RESULTS OF QMSA ANALYSIS}

The distributions of electron partial conductivity $\sigma_{n}$ vs mobility $\mu$ for different Sn concentrations $x$ are given in Fig. 29 .

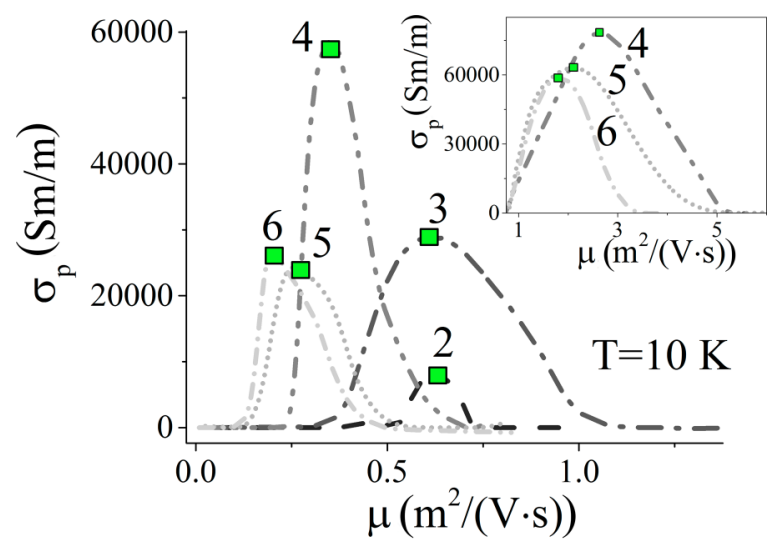

(a)

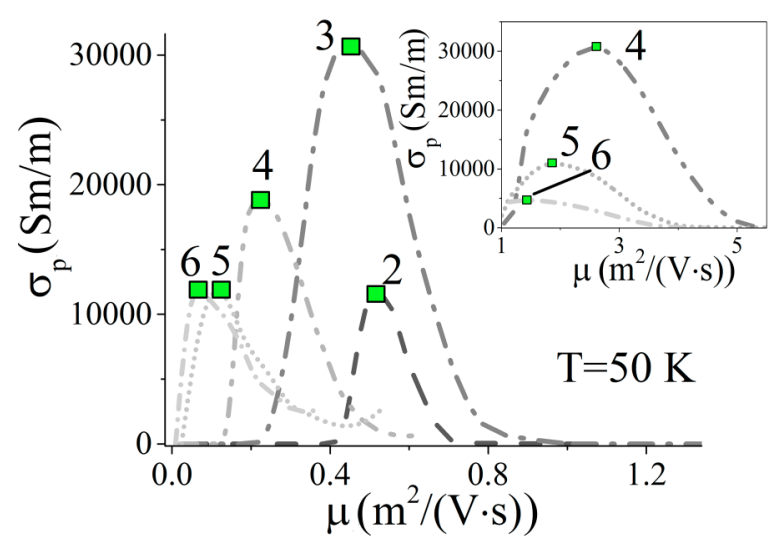

(b)

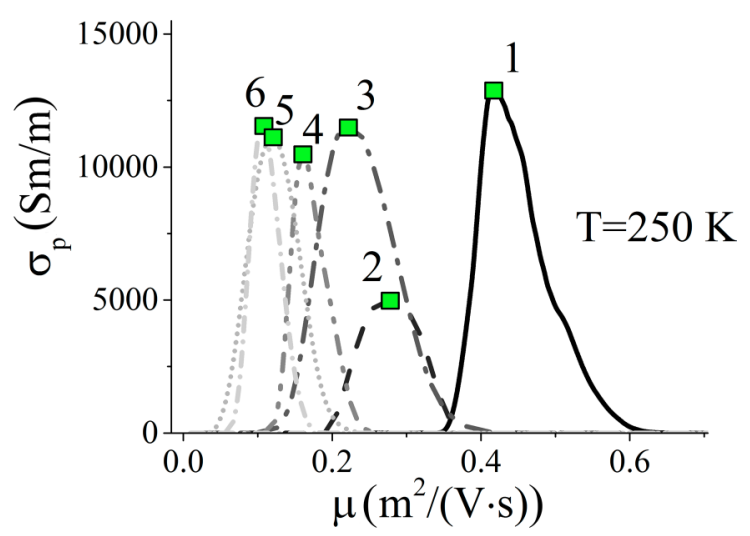

(c)

FIG. 30. Distribution of $T$-point hole partial conductivity $\sigma_{p}(\mu)$ of $\mathrm{Bi}_{100-x} \mathrm{Sn}_{x}$ vs mobility $\mu$. Different temperatures: $100 \mathrm{~K}(\mathrm{a}), 200 \mathrm{~K}$ (b), $300 \mathrm{~K}$ (c). Number marks $x$ (at.\%) of Sn: 1 (0) (pure Bi), 2 (0.01), 3 (0.02), 4 (0.04), 5 (0.06), 6 (0.08). Green squares mark centers of peaks. Insets: distributions of $L$-point hole partial conductivity $\sigma_{p}(\mu)$. 
The position of the peak corresponds to the mean mobility $\mu_{n}$ of the charge carrier. The area under the peak shows the magnitude of the carrier's contribution to the conductivity. The mobility of electrons is highly dependent on $\mathrm{Sn}$ concentration at lower temperatures [Fig. 29(a)]. With rise of temperature the dependence of electron mobilities on $\mathrm{Sn}$ concentration becomes much weaker [Figs. 29(b) and 29(c)]. The size of area under peaks decreases with $x$ on Fig. 29 which means that contribution of electrons to conductivity lowers.

The reason of small growth of electron mobility $\mu_{n}$ with $x$ may be explained by the nonparabolicity of the dispersion law near the $L$ point. Heremans and Hansen adapted the expression for mobility of charge carriers with pseudoparabolic dispersion law to Bi [19]:

$$
\mu(E)=\frac{q \tau_{0}}{m^{*}}\left(\frac{k_{B} T}{\left(1+E / E_{g}\right) E}\right)^{1 / 2},
$$

where $m^{*}$ is the effective mass, $E_{g}$ is the band gap at the $L$ point, and $\tau_{0}$ is a constant, which does not depend on the energy.

With increase of tin concentration $x$ the Fermi level shifts down from the upper $L$-point band to the band gap and to the lower $L$-point band. The average energy of electrons in more doped system therefore will be lower than in less doped. This may lead to increase of mobility according to Eq. (E1). When mobility of electrons is high enough (low temperatures) in doped samples, tin atoms act as mean-free path limiters so doping decreases mobility. At higher temperatures, phonon scattering plays a more important role so Eq. (E1) can be used to estimate mobilities behavior.

The distributions of hole partial conductivity $\sigma_{p}$ vs mobility $\mu$ are given in Fig. 30. There are two types of peaks. First are the peaks at lower mobilities which exist in all ranges of temperature and correspond to the heavy $T$-point holes. The others are peaks at higher mobilities visible under low temperatures for samples with the Sn doping $x>0.04$ at.\% [insets in Figs. 30(a) and 30(b)]. The last correspond to the $L$-point light holes.

The areas under $L$-point hole peaks remain similar with the temperature (Fig. 30). The area under the $L$-point peak quickly decreases with the temperature and completely disappear

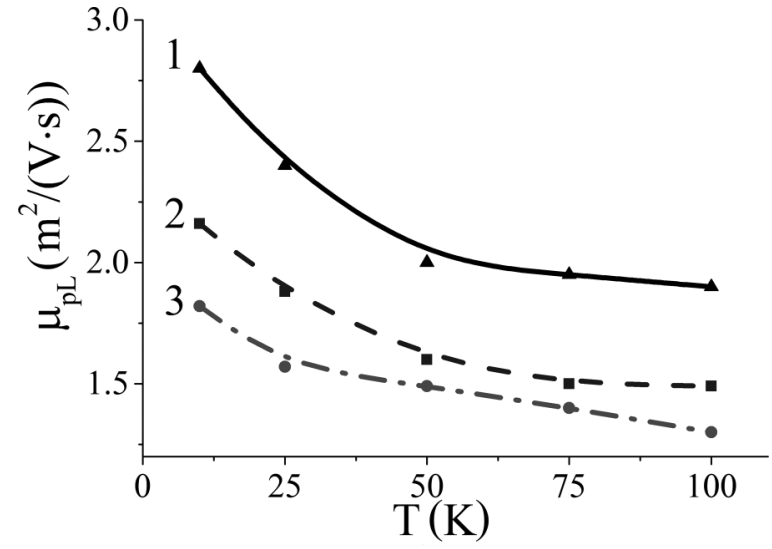

FIG. 31. Temperature dependencies of $L$-point hole mobility for different Sn concentration $x: 1(0.04)$ at.\%, $2(0.06)$ at.\%, $3(0.08)$ at. $\%$.

under room conditions. $T$-point hole mobility $\mu_{p}$ temperature dependencies are given in Fig. 31.

\section{APPENDIX F: SUBSTITUTION OF ELLIPTICAL DENSITY OF STATES TO SOMMERFELD EXPANSION}

The Lax density of states for elliptical band [20]

$$
g_{L}(E)=\frac{8 \pi \sqrt{2}}{h^{3} m^{*(3 / 2)}}\left(1+\frac{2 E}{E_{g}}\right) \sqrt{E\left(1+\frac{E}{E_{g}}\right)} .
$$

We substitute it to Eq. (5):

$$
\zeta(T)=\zeta_{0}-\frac{\pi^{2}}{6}\left(k_{B} T\right)^{2} \frac{g^{\prime}\left(\zeta_{0}\right)}{g\left(\zeta_{0}\right)}-\frac{7 \pi^{4}}{360}\left(k_{B} T\right)^{4} \frac{g^{\prime \prime \prime}\left(\zeta_{0}\right)}{g\left(\zeta_{0}\right)}-\cdots .
$$

The resulting expression for the temperature dependence of Fermi level will be

$$
\begin{aligned}
\zeta(T)= & \zeta_{0}-\frac{k_{B}^{2} \pi^{2} T^{2}\left(E_{g}^{2}+8 E_{g} \zeta_{0}+8 \zeta_{0}^{2}\right)}{12 \zeta_{0}\left(E_{g}+\zeta_{0}\right)\left(E_{g}+2 \zeta_{0}\right)} \\
& -\frac{7 E_{g}^{4} k_{B}^{4} \pi^{4} T^{4}}{960 \zeta_{0}^{3}\left(E_{g}+\zeta_{0}\right)^{3}\left(E_{g}+2 \zeta_{0}\right)}-\cdots
\end{aligned}
$$

The evaluations have shown that contribution of higher-order terms is negligible in a relevant range of parameters.
[1] H. Goldsmid, Adv. Phys. 14, 273 (1965).

[2] J. Chu and A. Sher, Physics and Properties of Narrow Gap Semiconductors (Springer, New York, 2008).

[3] F. Yue, S. Jit, and W. Hu, Sci. World J. 2014, 492521 (2014).

[4] J.-H. Bahk and A. Shakouri, Phys. Rev. B 93, 165209 (2016).

[5] S. Wang, J. Yang, T. Toll, J. Yang, W. Zhang, and X. Tang, Sci. Rep. 5, 10136 (2015).

[6] J. Mao, Z. Liu, and Z. Ren, npj Quantum Mater. 1 (2016).

[7] M. Alfaro-Gomez and E. Castro-Camus, Appl. Phys. Lett. 110, 042101 (2017).

[8] S. Ruffenach, A. Kadykov, V. V. Rumyantsev, J. Torres, D. Coquillat, D. But, S. S. Krishtopenko, C. Consejo, W. Knap, S. Winnerl et al., APL Mater. 5, 035503 (2017).
[9] J. Chu and A. Sher, Device Physics of Narrow Gap Semiconductors (Springer, New York, 2010).

[10] B. Cui, Y. Tang, and M. Grayson, in Quantum Sensing and Nanophotonic Devices XII, edited by M. Razeghi, E. Tournié, and G. J. Brown (SPIE, Bellingham, WA, 2015).

[11] T. Cunningham, Quantitative Mobility Spectrum Analysis of III-V Heterostructures on Silicon, Ph.D. thesis, Texas State University, 2012.

[12] B. Cui and M. Grayson, in Quantum Sensing and Nano Electronics and Photonics XIV, edited by M. Razeghi (SPIE, Bellingham, WA, 2017).

[13] R. D. Brown, R. L. Hartman, and S. H. Koenig, Phys. Rev. 172, 598 (1968). 
[14] S. Golin, Phys. Rev. 166, 643 (1968).

[15] B. Abeles and S. Meiboom, Phys. Rev. 101, 544 (1956).

[16] V. S. Edel'man, Zh. Eksp. Teor. Fiz. 68, 257 (1975) [Sov. Phys.JETP 41, 125 (1975)].

[17] S. H. Koenig, A. A. Lopez, D. B. Smith, and J. L. Yarnell, Phys. Rev. Lett. 20, 48 (1968).

[18] C. F. Gallo, B. S. Chandrasekhar, and P. H. Sutter, J. Appl. Phys. 34, 144 (1963).

[19] J. Heremans and O. P. Hansen, J. Phys. C: Solid State Phys. 12, 3483 (1979).

[20] D. Nakamura, M. Murata, H. Yamamoto, Y. Hasegawa, and T. Komine, J. Appl. Phys. 110, 053702 (2011).

[21] J. W. McClure, J. Low Temp. Phys. 25, 527 (1976).

[22] B. Lax, J. G. Mavroides, H. J. Zeiger, and R. J. Keyes, Phys. Rev. Lett. 5, 241 (1960).

[23] S. Kochowski and A. Opilski, Thin Solid Films 48, 345 (1978).

[24] O. Hansen, J. Heremans, and P. Lindelof, Solid State Commun. 42, 705 (1982).

[25] J. Heremans and O. P. Hansen, J. Phys. C: Solid State Phys. 16, 4623 (1983).

[26] L. C. Towle, A. Cybriwsky, and R. E. Stajdohar, J. Appl. Phys. 38, 668 (1967).

[27] G. Para, Thermomagnetic and thermoelectrical properties of nano- and microwires on the basis of Bi semimetal (Bi), Ph.D. thesis, Ghitu Institute of Electronic Engineering and Nanotechnologies, Chisinau, Moldova, 2010.

[28] A. A. Nikolaeva, D. V. Gitsu, T. E. Huber, L. A. Konopko, P. P. Bodiul, and Gh. Para, Rev. Adv. Mater. Sci. 8, 73 (2004).

[29] L. C. Hebel, Phys. Rev. 138, A1641 (1965).

[30] G. I. Leviev, V. B. Ikonnikov, and V. F. Gantmakher, Pis'ma Zh. Eksp. Teor. Fiz. 29, 634 (1979) [JETP Lett. 29, 580 (1979)].

[31] A. Nikolaeva, L. Konopko, T. Huber, and A. Tsurkan, J. Low Temp. Phys. 159, 258 (2010).
[32] Y. Hasegawa, Y. Ishikawa, T. Saso, H. Shirai, H. Morita, T. Komine, and H. Nakamura, Phys. B (Amsterdam) 382, 140 (2006).

[33] A. S. Fedotov, S. K. Poznyak, L. S. Tsybulskaya, V. G. Shepelevich, I. A. Svito, A. Saad, A. V. Mazanik, and A. K. Fedotov, Phys. Status Solidi B 252, 2000 (2015).

[34] Z. Dziuba and M. Górska, J. Phys. III France 2, 99 (1992).

[35] J. Antoszewski, D. J. Seymour, L. Faraone, J. R. Meyer, and C. A. Hoffman, J. Electron. Mater. 24, 1255 (1995).

[36] J. Antoszewski, L. Faraone, I. Vurgaftman, J. R. Meyer, and C. A. Hoffman, J. Electron. Mater. 33, 673 (2004).

[37] J. Meyer, C. Hoffman, F. Bartoli, J. Antoszewski, and L. Faraone, Quantitative mobility spectrum analysis for magnetic field dependent hall and resistivity data, US Patent No. 5,789,931 (1998).

[38] Liebermann, Rapidly Solidified Alloys: Processes-StructuresProperties-Applications (Materials Engineering) (CRC Press, Boca Raton, FL, 1993)

[39] O. V. Gusakova and V. G. Shepelevich, J. Surf. Invest.: X-Ray, Synchrotron, Neutron Technol. 5, 1011 (2011).

[40] E. Kuchis, Galvanomagnetic Effects and Methods of their Investigation, 3rd ed. (Radio i svyaz', Moscow, 1990).

[41] K. Seeger, Semiconductor Physics (Springer, Berlin, 2004).

[42] N. D. Mermin and N. W. Ashcroft, Solid State Physics (Thomson, India, 2003).

[43] W. A. Beck and J. R. Anderson, J. Appl. Phys. 62, 541 (1987).

[44] I. Vurgaftman, J. R. Meyer, C. A. Hoffman, D. Redfern, J. Antoszewski, L. Faraone, and J. R. Lindemuth, J. Appl. Phys. 84, 4966 (1998).

[45] J. W. McClure, Phys. Rev. 101, 1642 (1956).

[46] J. R. Meyer, C. A. Hoffman, F. J. Bartoli, D. A. Arnold, S. Sivananthan, and J. P. Fauri, Semicond. Sci. Technol. 8, 805 (1993). 